\title{
SedFoam: A multi-dimensional Eulerian two-phase model for sediment transport and its application to momentary bed failure
}

\author{
Zhen Cheng ${ }^{\mathrm{a}, *}$, Tian-Jian $\mathrm{Hsu}^{\mathrm{a}}$, Joseph Calantoni ${ }^{\mathrm{b}}$ \\ ${ }^{a}$ Civil and Environmental Engineering, University of Delaware, Newark, DE 19716, \\ U.S.A. \\ ${ }^{b}$ Marine Geosciences Division, Naval Research Laboratory, Stennis Space Center, MS \\ 39529, U.S.A.
}

\begin{abstract}
A multi-dimensional Eulerian two-phase model for sediment transport, called SedFoam, is presented. The model was developed under the open-source framework via the CFD toolbox OpenFOAM. With closures of particle stresses and fluid-particle interactions, the model is able to resolve processes in the concentrated region of sediment transport and hence does not require conventional bedload/suspended load assumptions. A modified $k-\epsilon$ closure was adopted for the carrier flow turbulence. The model was validated for Reynolds-averaged steady and oscillatory sheet flows and verified with empirical formulae for scour downstream of an apron. The model was used to study momentary bed failure (or plug flow) under sheet flow conditions. Model results revealed the existence of instabilities of the near-bed transport layer when momentary bed failure criteria was exceeded. These instabilities evolved into $5-10 \mathrm{~cm}$ billows and were responsible for the large transport
\end{abstract}

\footnotetext{
${ }^{*}$ Corresponding author

Email address: zcheng@udel.edu (Zhen Cheng)
}

Preprint submitted to Coastal Engineering

August 10, 2016

(C) 2016. This manuscript version is made available under the Elsevier user license http://www.elsevier.com/open-access/userlicense/1.0/ 
rate. The instabilities were associated with a large erosion depth, which was triggered by the combination of large bed shear stresses and large horizontal pressure gradients. Further numerical experiments confirmed the conjecture by previous studies that a criterion for onset of momentary bed failure in oscillatory sheet flow was a function of both the Shields parameter and Sleath parameter.

Keywords: two-phase, Eulerian-Eulerian, multi-dimensional, sediment transport, scour, bed instability

\section{1. Introduction}

2 The coastal zone is one of the most economically vibrant and culturally 3 diverse human habitats. However, it is vulnerable to sea-level rise and intense 4 storm events. Coastal morphological evolution is caused by a wide range of 5 coupled cross-shore and alongshore sediment transport processes driven by 6 waves, wave-induced currents and wave-breaking turbulence (e.g., Roelvink 7 et al., 2009). At operational forecasting scales, parameterizations of sediment 8 transport rate associated with these hydrodynamic forcing mechanisms are needed. Typically, sediment transport rate is parameterized by the bottom shear stress (e.g., Ribberink, 1998; van Rijn, 1984a), which works reasonably well for moderate wave conditions. However, during large wave events associated with intense storms, the predicted morphological evolution is less satisfactory (Ruessink and Kuriyama, 2008) and other mechanisms that are not parameterized by bottom shear stress may become dominant.

For example, laboratory experiments (Madsen, 1974; Sleath, 1999; Liu and Sato, 2005; Holway et al., 2012) and field observations (Conley and In- 
man, 1992; Foster et al., 2006) demonstrate that significant bed mobilization, called momentary bed failure or plug flow, may occur during the passage of steep waves. Although transport often occurs as sheet flow under steep waves, the timing of such large bed mobilization coincides with large horizontal pressure gradients. Moreover, momentary bed failure/plug flow can cause much larger transport than is predicted by typical sheet flow formula, and hence it is an essential process that should be included for the prediction of beach evolution during large wave events.

Our capability to resolve key mechanisms in the concentrated region of transport remains limited. Numerical models for coastal morphological evolution (Jacobsen et al., 2012; Warner et al., 2008; Lesser et al., 2004), local scour around structures (Hoffmans and Pilarczyk, 1995; Liu and Garcia, 2008) and bedform dynamics (Marieu et al., 2008; Chou and Fringer, 2010) typically separate the total sediment transport into bedload and suspended load components. Partitioning is necessary in order to efficiently model morphological evolution or to better resolve turbulence. However, bedload transport and erosion flux (pick up flux) are then parameterized semi-empirically (Ribberink, 1998; van Rijn, 1984c). Presently, these parameterizations do not explicitly include the effect of momentary bed failure.

On the other hand, the two-phase flow approach can resolve concentrated region of sediment transport by including closures of particle stresses and fluid-particle interactions in the governing equations. In the past decades, several two-phase numerical models have been developed, where the sediment phase is modeled either with an Eulerian scheme (e.g., Dong and Zhang, 1999, 2002; Hsu et al., 2004; Li et al., 2008; Amoudry and Liu, 2009; Bakhtyar et al., 
2010) or a Lagrangian scheme (e.g., Drake and Calantoni, 2001; Calantoni and Puleo, 2006; Calantoni and Thaxton, 2008; Heald et al., 2004). These two-phase models can resolve the full profiles of sediment transport without the need to divide the transport into bedload and suspended load components. For instance, one-dimensional-vertical (1DV) two-phase models are used to improve the parameterization of pickup flux in suspended load module under waves in sheet flow condition (Amoudry and Liu, 2010; Yu et al., 2012).

Due to the complexity of the model formulation, most of the existing two-phase models are based on the Reynolds-averaged approach and simplified into 1DV formulation. 1DV models cannot capture the development of inhomogeneous flow features (e.g., bedforms), flow instabilities (Charru and Mouilleron-Arnould, 2002) and scour (Amoudry and Liu, 2009). Hence, existing two-phase models are mainly restricted to sheet flow applications where the ensemble-averaged flow field is more or less fully developed in the streamwise and spanwise directions. Also, a model developed for 1DV cannot resolve turbulence, which is inherently three-dimensional. Amoudry and Liu (2009) developed a two-dimensional (2D) two-phase model to study scour downstream of an apron, and good agreements are obtained for the development of the $2 \mathrm{D}$ scour characteristics. A two-phase model has been developed by Bakhtyar et al. (2010) to study surf and swash zones sediment transport under the influence of wave breaking and wave runup/rundown. The model is shown to be capable of reproducing the observed beach profile evolution in the surf and swash zones qualitatively.

Motivated by this need, we have recently developed a multi-dimensional 
model for sediment transport that solves the complete Eulerian two-phase equations following the mathematical formulation similar to that of Hsu et al. (2004) and Yu et al. (2012). The mathematical formulation is solved numerically using the open-source CFD toolbox, OpenFOAM. OpenFOAM includes several multiphase flow modeling capabilities, such as bubbly flow (Rusche, 2002). Specifically, a two-phase flow solver in OpenFOAM called, twoPhaseEulerFoam (Rusche, 2002; Weller, 2002; Passalacqua and Fox, 2011) was adopted as the baseline. The numerical stability of the solver was improved and additional capabilities needed for coastal sediment transport applications were implemented. This new sediment transport model is called SedFoam. Although the numerical model was written in three spatial dimensions, the current version of the model has only been validated/verified for 2DV Reynolds-averaged condition.

The purpose of this study is to describe this new multi-dimensional model, SedFoam, its validation and its application to investigate momentary bed failure. The mathematical formulation of the model is discussed in Section 2. The numerical methodology is discussed in Section 2.1 and 2.2. Model validation for the present 2DV Reynolds-averaged formulation is presented in Section 3. The numerical investigation of momentary bed failures/plug flows is presented in Section 4, and concluding remarks are given in Sections 5 , respectively.

\section{Model Formulation}

Drew (1983) derives the Eulerian two-phase flow model by averaging over carrier fluid and dispersed particles. The resulting 3D governing equations 
for Eulerian two-phase flow can be considered as the counterpart of the clear fluid Navier-Stokes equations. Namely, the turbulent motions due to particleparticle interaction and particle-fluid interaction at the grain scale are parameterized via fluid stress, particle stress and inter-phase momentum transfer terms in the Eulerian two-phase equations. In order to efficiently apply these equations to turbulent flow, in which turbulent motions are generated by flow shear much larger than the grain scale, additional turbulence averaging or filtering is necessary. Through Reynolds-averaging, Hsu et al. (2004) developed the turbulence-averaged Eulerian two-phase model and simplified the model to 1DV to study sheet flow sediment transport. In this study, we adopted similar turbulence-averaged governing equations and closures to develop the numerical model in 3D.

\subsection{Governing equations}

Assuming that there is no mass transfer between the two phases, the mass conservation equations for fluid phase and sediment phase can be written as,

$$
\begin{gathered}
\frac{\partial(1-\phi)}{\partial t}+\frac{\partial(1-\phi) u_{i}^{f}}{\partial x_{i}}=0 \\
\frac{\partial \phi}{\partial t}+\frac{\partial \phi u_{i}^{s}}{\partial x_{i}}=0
\end{gathered}
$$

where $\phi$ is the sediment volumetric concentration, $u_{i}^{f}$ is the fluid velocity, and $u_{i}^{s}$ is the sediment velocity. In this study, $i=1,2,3$ represents streamwise, spanwise and vertical components, respectively. The momentum equations for fluid and particle phases are expressed as,

$$
\begin{gathered}
{ }_{113} \frac{\partial \rho^{f}(1-\phi) u_{i}^{f}}{\partial t}+\frac{\partial \rho^{f}(1-\phi) u_{i}^{f} u_{j}^{f}}{\partial x_{j}}=\begin{array}{c}
-\frac{\partial(1-\phi) p^{f}}{\partial x_{i}}+(1-\phi) f_{i}+\frac{\partial \tau_{i j}^{f}}{\partial x_{j}} \\
+\rho^{f}(1-\phi) g \delta_{i 3}+M_{i}^{f s},
\end{array}
\end{gathered}
$$


and

$$
\frac{\partial \rho^{s} \phi u_{i}^{s}}{\partial t}+\frac{\partial \rho^{s} \phi u_{i}^{s} u_{j}^{s}}{\partial x_{j}}=-\frac{\partial \phi p^{f}}{\partial x_{i}}+\phi f_{i}-\frac{\partial p^{s}}{\partial x_{i}}+\frac{\partial \tau_{i j}^{s}}{\partial x_{j}}+\rho^{s} \phi g \delta_{i 3}+M_{i}^{s f}
$$

where $\rho^{f}$ and $\rho^{s}$ are fluid and sediment density, respectively, $g_{i}$ is the gravitational acceleration and $p^{f}$ is the fluid pressure. The external pressure gradient that drives the flow is written as $f_{i}$. The fluid stress $\tau_{i j}^{f}$ includes the grain-scale (viscous) stress and the turbulent Reynolds stress, which are calculated with a two-equation turbulence model. The particle normal stress $p^{s}$ and shear stress $\tau_{i j}^{s}$ are modeled with the kinetic theory of granular flow and a phenomenological closure of frictional contact stresses. Finally, $M_{i}^{f s}$ and $M_{i}^{s f}$ represent the inter-phase momentum transfer between fluid and particle phase, and $M_{i}^{f s}=-M_{i}^{s f}$ follows the Newton's 3rd law.

\subsection{Closure Models}

\subsubsection{Inter-phase Momentum Transfer}

In the two-phase flow formulation, the momentum exchange of the fluid phase and particle phase are coupled through the inter-phase momentum transfer terms (Maxey and Riley, 1983). For simplicity, only the dominant terms, namely the drag force and the buoyancy force are included in the present study. Thus the momentum exchange has the following form,

$$
M_{i}^{f s}=-\phi \beta\left(u_{i}^{f}-u_{i}^{s}\right)+\beta \frac{\nu^{f t}}{\sigma_{c}} \frac{\partial \phi}{\partial x_{i}}+p^{f} \frac{\partial(1-\phi)}{\partial x_{i}} .
$$

The first two terms on the right-hand-side (RHS) are due to drag force with $\beta$ as the drag parameter. The first term represents averaged drag force due to mean velocity difference between fluid and particle phases. The second 
term is the fluid turbulent suspension term, which results from the correlation of sediment concentration and fluid velocity fluctuations. The gradient transport assumption was adopted here with a closure for $\nu^{f t}$ representing the turbulent viscosity, which was calculated with a turbulence closure (see Section 2.2.2), and $\sigma_{c}$ is the Schmidt number (see Table 1). The Schmidt number is a ratio of fluid turbulent viscosity to the sediment diffusivity, and according to previous studies, values smaller than unity were reported (Coleman, 1970; van Rijn, 1984b; Amoudry et al., 2005; Revil-Baudard et al., 2015). However, there does not seem to be a universally agreed value or relationship for Schmidt number for different sediment properties and flow conditions. In the present model, the Schmidt number is chosen to be 1 for simplicity, and its effect on the model results will be briefly discussed (see Section 3.1). The last term on the RHS of Eq. (5) represents the averaged buoyancy force. The correlation term between sediment concentration and fluid pressure is ignored in the turbulence averaged momentum equations. However, in the closure of eddy viscosity, the effect is included via buoyancy terms in the $k-\epsilon$ equations.

For the closure of the drag parameter $\beta$, we follow Ding and Gidaspow (1990), who combined the model of Ergun (1952) for dense sediment concentration $(\phi \geq 0.2)$ and the model of Wen and Yu (1966) for lower sediment concentration $(\phi<0.2)$,

$$
\beta=\left\{\begin{array}{c}
\frac{150 \phi \nu^{f} \rho^{f}}{(1-\phi) d^{2}}+\frac{1.75 \rho^{f}\left|u^{f}-u^{s}\right|}{d}, \phi \geq 0.2 \\
\frac{0.75 C_{d} \rho^{f}\left|u^{f}-u^{s}\right|(1-\phi)^{-1.65}}{d}, \phi<0.2
\end{array},\right.
$$

where $d$ is the sediment diameter, and the drag coefficient $C_{d}$ is calculated 
as:

$$
C_{d}=\left\{\begin{array}{c}
\frac{24\left(1+0.15 R e_{p}^{0.687}\right)}{R e_{p}}, R e_{p} \leq 1000 \\
0.44, R e_{p}>1000
\end{array},\right.
$$

in which, $R e_{p}=(1-\phi)\left|u^{f}-u^{s}\right| d / \nu^{f}$ is the particle Reynolds number, and $\nu^{f}$ is the fluid molecular viscosity.

\subsubsection{Fluid turbulence Model}

Because the present model equations were obtained by averaging over turbulence, the fluid stresses consist of a large-scale component $R_{i j}^{f t}$ (i.e., Reynolds stress) and a grain-scale component $r_{i j}^{f}$, which includes the viscous stress and an additional effect due to fluid-particle interaction at grain scale. In the present study, this later effect is ignored and only viscous stress is considered. Thus, the total fluid stress is written as,

$\tau_{i j}^{f}=R_{i j}^{f t}+r_{i j}^{f}=\rho^{f}(1-\phi)\left[\left(\nu^{f t}+\nu^{f}\right)\left(\frac{\partial u_{i}^{f}}{\partial x_{j}}+\frac{\partial u_{j}^{f}}{\partial x_{i}}-\frac{2}{3} \frac{\partial u_{k}^{f}}{\partial x_{k}} \delta_{i j}\right)-\frac{2}{3} k^{f} \delta_{i j}\right]$.

The turbulent eddy viscosity $\nu^{f t}$ is calculated by the fluid turbulence kinetic energy $k^{f}$ and turbulent dissipation rate $\epsilon^{f}$,

$$
\nu^{f t}=C_{\mu} \frac{\left(k^{f}\right)^{2}}{\epsilon^{f}},
$$

where $C_{\mu}$ is an empirical coefficient (see Table 1). The turbulent kinetic energy $k^{f}$ (TKE) is calculated by its balance equation appropriate for sand particles in water (Yu et al., 2010),

$$
\begin{aligned}
& { }_{178} \frac{\partial k^{f}}{\partial t}+u_{j}^{f} \frac{\partial k^{f}}{\partial x_{j}}=\frac{R_{i j}^{f t}}{\rho^{f}} \frac{\partial u_{i}^{f}}{\partial x_{j}}+\frac{\partial}{\partial x_{j}}\left[\left(\nu^{f}+\frac{\nu^{f t}}{\sigma_{k}}\right) \frac{\partial k^{f}}{\partial x_{j}}\right]-\epsilon^{f}-\frac{2 \beta(1-\alpha) \phi k^{f}}{\rho^{f}(1-\phi)} \\
& -\frac{1}{(1-\phi)} \frac{\nu^{f t}}{\sigma_{c}} \frac{\partial \phi}{\partial x_{j}}(s-1) g_{j} \text {. }
\end{aligned}
$$


Except for the last two terms on the RHS, the rest of terms in the present $k^{f}$ equation are similar to those in the clear fluid TKE equation. The last term in Eq. (10) represents the buoyancy effect. For typical sediment concentration with an upward decaying profile, the buoyancy term represents the wellknown sediment-induced density stratification that can attenuate turbulence. The fourth term on the RHS represents attenuation of TKE due to drag. The parameter $\alpha$ is proposed to characterize the degree that particles follow the fluid velocity fluctuations and it can be quantified by the Stokes number $S t=t_{p} / t_{l}$ (Balachandar and Eaton, 2010), where $t_{p}=\rho^{s} / \beta$ is the particle response time, and $t_{l}=k^{f} /\left(6 \epsilon^{f}\right)$ is the characteristic time scale of energetic eddies. Considering a given particle encounters a turbulent eddy on its path, if the particle has very small inertia $(S t \ll 1)$, it can closely follow the eddy motion. However, if $S t \sim O(1)$, the particle cannot completely follow the eddy motion and may cause disturbance to the eddy. Finally, if $S t \gg 1$, the particle trajectory is hardly affected by the eddy motion. With these observations, Danon et al. (1977) and Chen and Wood (1985) proposed an exponential function for $\alpha$,

$$
\alpha=e^{-B \cdot S t},
$$

where $B$ is an empirical coefficient. It is evident from the model that the fourth term in Eq. (10) represents a dissipation mechanism for the turbulent kinetic energy. It is also possible that the presence of particle provides a generation mechanism of flow turbulence. However, this is observed for particles with very large Stokes number. For sand transport in water considered in this study, this turbulence generation mechanism is unlikely and it is neglected. 
Finally, the balance equation for the rate of turbulent kinetic energy dissipation $\epsilon^{f}$ is written as,

$$
\begin{gathered}
\frac{\partial \epsilon^{f}}{\partial t}+u_{j}^{f} \frac{\partial \epsilon^{f}}{\partial x_{j}}=C_{1 \epsilon} \frac{\epsilon^{f}}{k^{f}} \frac{R_{i j}^{f t}}{\rho^{f}} \frac{\partial u_{i}^{f}}{\partial x_{j}}+\frac{\partial}{\partial x_{j}}\left[\left(\nu^{f}+\frac{\nu^{f t}}{\sigma_{\epsilon}}\right) \frac{\partial \epsilon^{f}}{\partial x_{j}}\right]-C_{2 \epsilon} \frac{\epsilon^{f}}{k^{f}} \epsilon^{f} \\
-C_{3 \epsilon} \frac{\epsilon^{f}}{k^{f}} \frac{2 \beta(1-\alpha) \phi k^{f}}{\rho^{f}(1-\phi)}-C_{4 \epsilon} \frac{\epsilon^{f}}{k^{f}} \frac{1}{(1-\phi)} \frac{\nu^{f t}}{\sigma_{c}} \frac{\partial \phi}{\partial x_{j}}(s-1) g_{j} .
\end{gathered}
$$

As discussed in Hsu et al. (2004), due to lack of comprehensive experimental data, the coefficients associated with the present two-equation closure are adopted from their clear fluid counterpart. The coefficient $C_{3 \epsilon}$ in Eq. (12) is chosen to be 1.2. For the coefficient associated with the buoyancy term, $C_{4 \epsilon}=0$ is used in stably stratified condition, while it is set to 1 for unstably stratified condition. Table 1 summarizes the coefficients used for the present turbulence closure. The coefficient $B$ in Eq. (11) is left as the only free model parameter to be calibrated with measured data.

Table 1: List of coefficients in fluid turbulence closure.

\begin{tabular}{cccccccc}
\hline$C_{\mu}$ & $C_{1 \epsilon}$ & $C_{2 \epsilon}$ & $C_{3 \epsilon}$ & $C_{4 \epsilon}$ & $\sigma_{k}$ & $\sigma_{\epsilon}$ & $\sigma_{c}$ \\
\hline 0.09 & 1.44 & 1.92 & 1.2 & 0 or 1.0 & 1.0 & 1.3 & 1.0 \\
\hline
\end{tabular}

\subsubsection{Particle Stress Closure}

In this study, the closure of particle stresses includes two components. For low to moderate sediment concentration, inter-granular interaction is assumed to be dominated by binary collisions and a closure based on kinetic theory is adopted. For large sediment concentration, binary collision becomes unlikely and inter-granular interaction is dominated by enduring 
contact/frictional forces among particles (Tardos, 1997). For the present sediment transport application where the immobile bed and concentrated region of transport are also simulated (Hsu et al., 2004), we assume that particle pressure and particle stress both consist of a collisional-kinetic component (super-script 'sc') and a frictional component (super-script 'sf') (Johnson and Jackson, 1987),

$$
\begin{gathered}
p^{s}=p^{s c}+p^{s f}, \\
\tau_{i j}^{s}=\tau_{i j}^{s c}+\tau_{i j}^{s f} .
\end{gathered}
$$

The closure of the collisional-kinetic component is based on the kinetic theory of granular flow where particle stress and particle pressure are quantified by the granular temperature, $\Theta$, which is defined as one-third of the kinetic energy of particle velocity fluctuation. Similar to fluid TKE, balance equation of granular temperature can be derived, but a closure model is needed. In this study, we adopt the model of kinetic theory of granular flow. The model is originally developed for dry granular flow consists of smooth, slightly inelastic, spherical particles (Jenkins and Savage, 1983; Lun and Savage, 1987). Here, we implement the model suggested by Ding and Gidaspow (1990), which takes into account of the fluid phase. The balance equation for granular temperature is written as,

$$
\frac{3}{2}\left[\frac{\partial \phi \rho^{s} \Theta}{\partial t}+\frac{\partial \phi \rho^{s} u_{j}^{s} \Theta}{\partial x_{j}}\right]=\left(-p^{s c} \delta_{i j}+\tau_{i j}^{s c}\right) \frac{\partial u_{i}^{s}}{\partial x_{j}}-\frac{\partial q_{j}}{\partial x_{j}}-\gamma_{s}+J_{i n t},
$$

where the terms on the RHS are the production of granular temperature, the flux of granular temperature $q_{j}$, the energy dissipation rate due to inelastic collision $\gamma_{s}$ and the production (or dissipation) due to the interaction with the carrier fluid phase $J_{\text {int }}$. Following Ding and Gidaspow (1990), closure of 
particle pressure is written as,

$$
p^{s c}=\rho^{s} \phi\left[1+2(1+e) \phi g_{s 0}\right] \Theta
$$

where $e$ is the coefficient of restitution during collision. The radial distribution function $g_{s 0}$ is introduced to describe the crowdiness of particle, which can be calculated as (Carnahan and Starling, 1969),

$$
g_{s 0}=\frac{2-\phi}{2(1-\phi)^{3}}
$$

The radial distribution function $g_{s 0}$ is a sharp increasing function of sediment concentration, $\phi$, representing more frequent collisions, which further causes larger particle pressure. It has been demonstrated that the formula of Carnahan and Starling (1969) under-predicts $g_{s 0}$ when concentration $\phi>0.57$ (Ding and Gidaspow, 1990; Sinclair and Jackson, 1989). However, it is noted that in the present study when $\phi>0.57$, inter-granular interactions are dominated by enduring contact/frictional component of the stress. In fact, granular temperature modeled by the kinetic theory reduces to nearly zero when particle concentration is approaching $\phi=0.57$. Therefore, we still adopt the radial distribution function of Carnahan and Starling (1969) for simplicity.

Following Gidaspow (1994), the particle stress is calculated as,

$$
\tau_{i j}^{s c}=\mu^{s c}\left[\frac{\partial u_{i}^{s}}{\partial x_{j}}+\frac{\partial u_{j}^{s}}{\partial x_{i}}\right]+\left(\lambda-\frac{2}{3} \mu^{s c}\right) \frac{\partial u_{k}^{s}}{\partial x_{k}} \delta_{i j} .
$$

Through the kinetic theory, the particle shear viscosity is calculated as a function of granular temperature and radial distribution function,

${ }_{269} \quad \mu^{s c}=\rho^{s} d \sqrt{\Theta}\left[\frac{4}{5} \frac{\phi^{2} g_{s 0}(1+e)}{\sqrt{\pi}}+\frac{\sqrt{\pi} g_{s 0}(1+e)(3 e-1) \phi^{2}}{15(3-e)}+\frac{\sqrt{\pi} \phi}{6(3-e)}\right]$. 
Similarly, the bulk viscosity is calculated as,

$$
\lambda=\frac{4}{3} \phi^{2} \rho^{s} d g_{s 0}(1+e) \sqrt{\frac{\Theta}{\pi}} .
$$

It is worth mentioning that the sediment collisional stress closure adopted in this study differs from that used in Hsu et al. (2004). In the present model, the kinetic contribution of particle stress is included, thus the sediment stress closure can be extended to very dilute condition without an artificial cut-off as discussed in Hsu et al. (2004). The modification was essential for numerical stability of the model when extending to multi-dimensions. Meanwhile, the sediment collisional stress closure adopted here takes into account of the effect inelastic collisions, which is more appropriate for sand transport in water. The closure of granular temperature flux is assumed to be analogous to the Fourier's law of conduction,

$$
q_{j}=-\kappa^{s c} \frac{\partial \Theta}{\partial x_{j}}
$$

where the $\kappa^{s c}$ is the conductivity of granular temperature, calculated as,

$$
\kappa^{s c}=\rho^{s} d \sqrt{\Theta}\left[\frac{2 \phi^{2} g_{s 0}(1+e)}{\sqrt{\pi}}+\frac{9 \sqrt{\pi} g_{s 0}(1+e)^{2}(2 e-1) \phi^{2}}{2(49-33 e)}+\frac{5 \sqrt{\pi} \phi}{2(49-33 e)}\right] .
$$

The dissipation rate due to inelastic collision is calculated based on that proposed by Ding and Gidaspow (1990),

$$
\gamma_{s}=3\left(1-e^{2}\right) \phi^{2} \rho^{s} g_{s 0} \Theta\left[\frac{4}{d}\left(\frac{\Theta}{\pi}\right)^{1 / 2}-\frac{\partial u_{j}^{s}}{\partial x_{j}}\right] .
$$

Due to the presence of carrier fluid phase, carrier flow turbulence can also induce particle fluctuations. Following Hsu et al. (2004), the fluid-particle interaction term is given as,

$$
J_{\text {int }}=\phi \beta\left(2 \alpha k^{f}-3 \Theta\right) .
$$


312

When the volumetric concentration of particles becomes close to random loose packing $(\phi \approx 0.57)$, particles are constantly in contact with one another, and most of the particle phase energy is dissipated by friction between sliding particles, rather than inelastic collision (Tardos, 1997). In this condition, the normal stress acts to support the particles and the sediment bed behaves like a solid. In other words, when the sediment concentration exceeds certain threshold value $\phi_{f}$, sediment transport is in the stage of enduring contact, kinetic theory is no longer valid, and a frictional stress model needs to be incorporated. In the 1DV model of Hsu et al. (2004), the location of immobile bed was dynamically tracked using a Hertz contact law combined with a Coulomb criteria while modeling the sediment phase transport above the instantaneous immobile bed. Our experience suggested that extending this formulation to multi-dimensions was not practical. Consequently, in the present model, we adopt a different approach aimed at modeling the full transport of sediment phase into the immobile bed. The particle stresses due to the enduring contact in the concentrated regions are modeled with a phenomenological closure that ensures a solid-like behavior when sediment concentration is near 0.6 without the need to track the location of the immobile bed. Following Johnson and Jackson (1987), the enduring contact component of particle pressure is calculated as,

$$
p^{s f}=\left\{\begin{array}{r}
0, \phi<\phi_{f} \\
F \frac{\left(\phi-\phi_{f}\right)^{m}}{\left(\phi_{\max }-\phi\right)^{n}}, \phi \geq \phi_{f},
\end{array}\right.
$$

where $\phi_{f}=0.57, \phi_{\max }=0.635$ and $F, m$ and $n$ are empirical coefficients to be discussed next. The particle stress due to frictional contact is calculated 
by,

$$
\tau_{i j}^{s f}=-2 \mu^{s f} S_{i j}^{s},
$$

where $\mu^{s f}$ is the frictional viscosity and $S_{i j}^{s}$ is the deviatoric part of strain rate tensor of sediment phase,

$$
S_{i j}^{s}=\frac{1}{2}\left(\frac{\partial u_{i}^{s}}{\partial x_{j}}+\frac{\partial u_{j}^{s}}{\partial x_{i}}\right)-\frac{1}{3} \frac{\partial u_{k}^{s}}{\partial x_{k}} \delta_{i j}
$$

Srivastava and Sundaresan (2003) combine the frictional normal stress from Johnson and Jackson's model and the frictional viscosity from Schaeffer (1987) model, and suggest the friction viscosity to be calculated by,

$$
\mu^{s f}=\frac{\sqrt{2} p^{s f} \sin \left(\theta_{f}\right)}{2 \sqrt{S_{i j}^{s} \cdot S_{i j}^{s}}},
$$

where $\theta_{f}$ is the angle of repose and is taken to be $\theta_{f}=28^{\circ}$ for sand.

This frictional shear viscosity model has the capability to capture the transition of solid-like feature to fluid-like feature of the sediment bed. In the original model of Srivastava and Sundaresan (2003), a correction of $\Theta / d^{2}$ is added to the denominator of Eq. (28) for numerical stability. However, we found that in the present sediment transport application, the shear rate in the concentrated region of transport was rather small. Therefore, the correction of $\Theta / d^{2}$ can introduce large error. In this study, this extra term was replaced by a small number of $10^{-20}$ to ensure numerical stability. In sediment transport, the frictional components of particle pressure and particle stress play a definite role to ensure the existence of an immobile sediment bed and above which, a low mobility layer of enduring contact can be modeled (Hsu et al., 2004). Hence, the empirical coefficients in Eq. (25) were calibrated to ensure a stable sediment bed can be established. The following 
values are adopted,

$$
F=0.05, m=3, n=5 .
$$

With this new frictional stress model and choice of empirical coefficients, the sediment concentration becomes close but rarely exceeds the maximum packing limit of 0.635. Notice that in the original model of Johnson and Jackson (1987) applied to debris flow, these coefficients are suggested to be $F=0.05, m=2$ and $n=5$. Hence, the present model applied to sediment transport in a bottom boundary layer also gives similar values.

\subsection{Numerical Implementation}

The numerical implementation of the present model utilizes the opensource CFD toolbox OpenFOAM, which provides a variety of finite volume method libraries and solvers. OpenFOAM includes several single phase and multiphase flow solvers. Specifically, the mathematical formulations presented above were implemented by modifying a two-phase flow solver, twoPhaseEulerFoam (Rusche, 2002; Weller, 2002; Passalacqua and Fox, 2011).

The solution procedure is outlined in the Fig. 1, and the sequence of solution is summarized as follow: At beginning of the numerical simulation, the sediment concentration, fluid and sediment velocity, fluid turbulence and granular temperature are initialized in a smooth manner. In each computational cycle, the sediment concentration is first updated, followed by the drag coefficient, $\beta$. The fluid turbulence and granular temperatures are then solved, and the fluid viscosity and sediment stresses are updated. For the velocity-pressure coupling procedure, the standard PIMPLE approach, which is a merged PISO-SIMPLE algorithm, is used after the version 2.1.0 of 


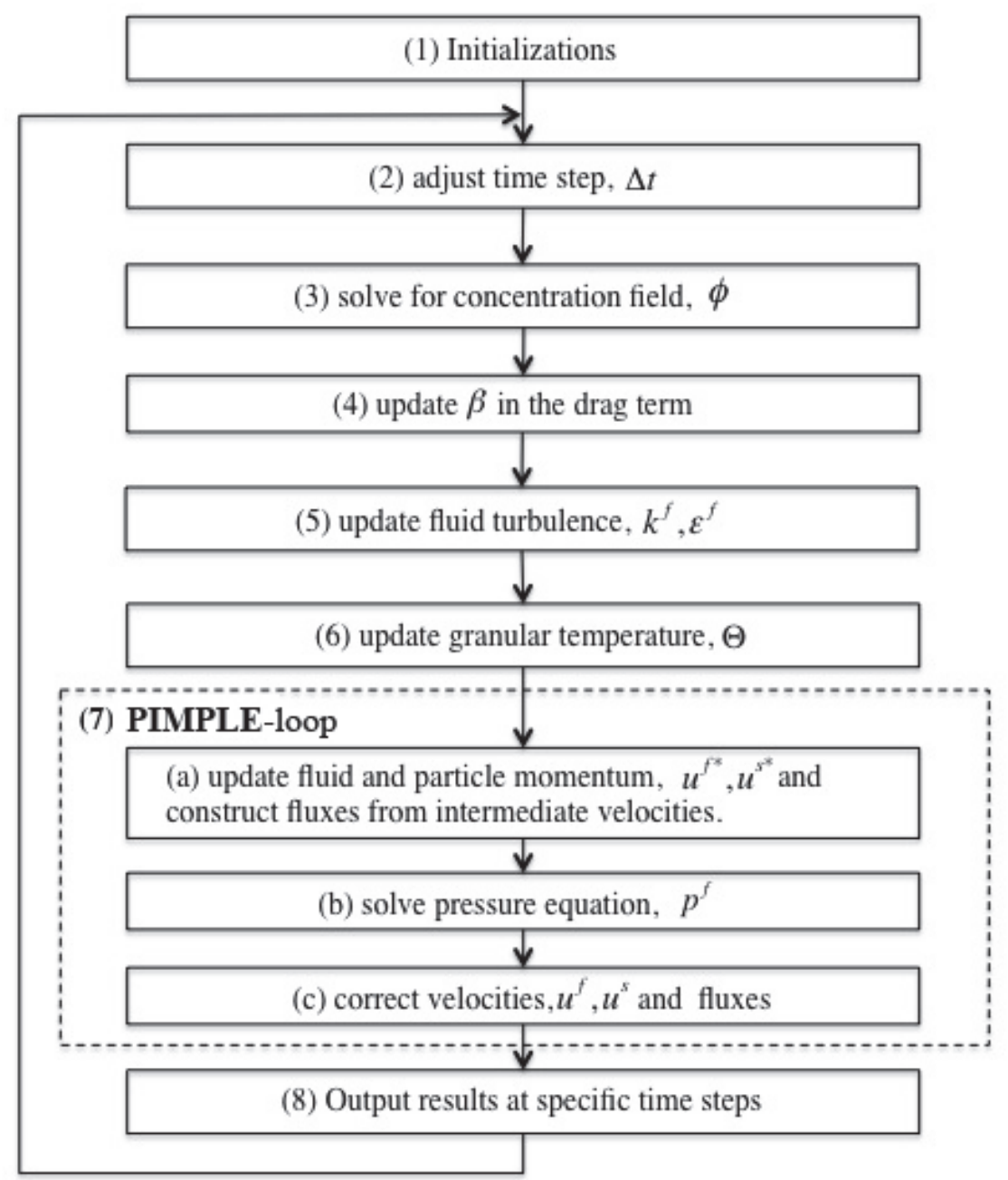

Fig. 1: Flow chart of solution procedure

OpenFOAM. The intermediate velocities are first solved using the momentum equation without the pressure gradient term. To ensure the Newton's third law in the momentum equations of the fluid phase and sediment phase, the drag force is calculated explicitly using the velocities at the previous time step. The pressure equation is constructed and solved by considering the continuity equations for both phases. In the present model, collocated 
variables are solved at cell centers, and the method of Rhie and Chow (1983) is adopted for solving the pressure equations to prevent velocity-pressure decoupling and oscillation in the pressure field. After solving the pressure, the fluid and sediment velocities are updated, and the resulting fluid phase and particle phase velocities both satisfy their mass conservation. The velocities are then used to update the volumetric sediment concentrations, turbulence quantities, fluid stresses, granular temperature and particle stresses.

The advection terms in the governing equations are solved using a secondorder TVD scheme, based on the Sweby limiter (Sweby, 1984). Two special versions of this scheme are available in OpenFOAM: (1) limitedLinearV, which is specifically for vectors, and the limiter is applied independently in each direction; (2) limitedLinear01, which ensures the scalar variable is bounded between 0 and 1, is adopted to solve the volumetric concentration $\phi$. In this manner, the convective terms are treated implicitly after being linearized using the value of the velocity flux at the previous iteration. The central difference schemes are used for diffusion terms, and the standard Crank-Nicolson scheme is used for time integration. The time step is updated every cycle to satisfy the Courant-Friedrichs-Lewy (CFL) condition and it is adjusted in a smooth fashion to ensure numerical stability. More details about the numerical implementations can be found in Cheng and Hsu (2014).

\section{Model Validation}

The model was first calibrated/validated with laboratory flume data of Sumer et al. (1996) for sand transport in steady channel flow under the sheet flow condition. Then, the calibrated model was further validated with 
measured sheet flow sediment concentration and flow velocity for 1DV/2DV oscillatory flow (O'Donoghue and Wright, 2004) and 2DV scour development downstream of an apron (Amoudry and Liu, 2009).

\subsection{Steady Sheet Flow}

The intensity of sediment transport is often quantified by the bed shear stress $\tau_{b}$. The non-dimensional bed shear stress, called the Shields parameter, is defined as, $\theta=\tau_{b} /\left(\rho^{s}-\rho^{f}\right) g d$. Compared with Hsu et al. (2004), the present model adopts a new turbulence modulation parameterization for $\alpha$ in the $k-\epsilon$ equations as well as in the granular temperature equation. The improved $\alpha$ parameterization model is expected to be more robust in predicting sediment suspension over a wider range of sediment size and density (Kranenburg et al., 2014). We first calibrate the model with fine sand $\left(d=0.13 \mathrm{~mm} ; \rho^{s}=2650 \mathrm{~kg} / \mathrm{m}^{3}\right)$ in the sheet flow condition driven by steady flows in a channel (Sumer et al., 1996). Specifically, the model coefficient $B$ in Eq. (11) associated with turbulence modulation due to sediment is calibrated and demonstrated to work well for Shields parameter ranging from 1.0 to 2.2 .

Table 2: A summary of the fine sand (grain diameter $d=0.13 \mathrm{~mm}$, specific gravity $s=2.65$ ) experiments reported in Sumer et al. (1996) used here for model calibration.

\begin{tabular}{cccc}
\hline Shields parameter $\theta$ & $\tau_{b}(\mathrm{~Pa})$ & $h(\mathrm{~cm})$ & $f_{x}(\mathrm{~Pa} / \mathrm{m})$ \\
\hline 1.10 & 2.31 & 10.5 & 52 \\
1.68 & 3.54 & 10.8 & 82 \\
2.20 & 4.63 & 11.2 & 110 \\
\hline
\end{tabular}

As shown in Table 2, the three different Shields parameters modeled here 
cover a range from $\theta=1.1$ to 2.2 . In the numerical simulation, both the flow depth and bottom shear stress were kept the same as Sumer et al. (1996). The flow was driven by a prescribed constant streamwise pressure gradient, which was determined by matching the targeted bottom shear stress at the bed. Since sheet flow is typically assumed to be statistically homogeneous in the streamwise and spanwise directions, 1DV simulations were carried out, i.e., $L_{x}$ and $L_{y}$ in the streamwise and spanwise directions are reduced to one grid cell (see Fig. 2). Periodic boundary conditions were used in the streamwise direction. For 2D simulation in the OpenFOAM framework, a dummy boundary condition called 'empty' can be used in the spanwise direction. Wall boundaries were imposed on both the top and bottom boundary of the computational domain. The total domain height is set to be $L_{z}=0.15 \mathrm{~m}$ with an initial sediment bed depth of $h_{b}=0.05 \mathrm{~m}$. We use 600 uniformly spaced grid points in the vertical direction, and the grid resolution is $\Delta z=0.25 \mathrm{~mm}$.

When using $B=0.22$, we obtained good agreement with measured sediment concentration profiles for Shields parameter in the range of $\theta=1.1$ to 2.2 for fine sand (Fig. 3(a),(e),(i)). Although the fluid and sediment velocities were not measured, the model results for the streamwise fluid velocity were shown in Fig. 3 (b),(f),(j). Due to the small particle size considered here, the difference between the fluid phase and sediment phase velocity was within $0.01 \mathrm{~m} / \mathrm{s}$ and hence the sediment phase velocities are not shown here for brevity. The model also provided profiles of fluid turbulent kinetic energy and granular temperature (see Fig. $3(\mathrm{c}),(\mathrm{g}),(\mathrm{k}))$. The velocity fluctuation energies were both zero in the bed, while the fluid turbulent kinetic energy increased rapidly to its peak value several millimeters above the bed due to 
large flow shear. Similar behavior can be observed for granular temperature. The fluid shear stress and particle shear stress are shown in Fig. 3 (d),(h),(l). It can be observed that the particle shear stress increased rapidly in the sheet layer and peaked at the immobile bed. On the other hand, the fluid shear stress dropped sharply in the sheet flow layer, and became close to zero at the immobile bed. The summation of particle shear stress and fluid (mostly turbulent) shear stress gave the expected linear profile of total shear stress in a steady channel flow. Note that the $B$ value was calibrated with a constant Schmidt number $S c=1$. Simulations showed that the $B$ value was also sensitive to the choice of Schmidt number. In order to produce similar sediment concentration profiles, the $B$ needed to be increased to $B=0.6$ when a Schmidt number of $S c=0.5$ was used. Here, the Schmidt number is assumed to be $S c=1$, and the calibrated value of $B=0.22$ will be used for the remainder of this paper.

\subsection{Oscillatory Sheet Flow}

O'Donoghue and Wright (2004) measured sediment concentration and flow velocity in the concentrated region of transport under a range of oscillatory sheet flow conditions. The experimental results have been used by many researchers to validate their models (e.g., Li et al., 2008; Chen et al., 2011; Yu et al., 2012). The calibrated model coefficient $B=0.22$ was used here to validate sheet flow of medium sand $(d=0.28 \mathrm{~mm})$ under oscillatory flow condition.

In the numerical model, the oscillatory flow is driven by a mean streamwise pressure gradient. Outside the wave boundary layer, the shear stress 
vanishes, and the momentum equation reduces to,

$$
f_{x}=\rho^{f} \frac{\partial U_{0}}{\partial t}
$$

where $U_{0}$ is the free stream velocity. Following O'Donoghue and Wright (2004), the asymmetric flow was forced by the second-order Stokes wave motion,

$$
U_{0}(t)=U_{1} \sin \left(\omega t+\sigma_{0}\right)-U_{2} \cos \left(2 \omega t+2 \sigma_{0}\right)
$$

where $U_{1}$ and $U_{2}$ are velocity amplitudes for each harmonic, $\omega=2 \pi / T$ is the oscillatory frequency, $T$ is the oscillatory period and $\sigma_{0}$ is the a phase lag, which is chosen such that the free-stream velocity is zero at $t=0$. The flow asymmetry is defined as, $a=U_{\max } /\left(U_{\max }-U_{\min }\right)$, in which $U_{\max }$ and $U_{\min }$ are maximum and minimum free-stream velocities, respectively. Here, we consider the asymmetric flow case with wave period $T=7.5 \mathrm{~s}$ and maximum velocity $U_{\max }=1.5 \mathrm{~m} / \mathrm{s}$ with $a=0.63$ (MA7515 in O'Donoghue and Wright 2004). A 1DV simulation was first carried out, and the domain height is $L_{z}=0.3 \mathrm{~m}$, with an initial sediment bed depth $h_{b}=0.05 \mathrm{~m}$. A total of 240 grid points are used in the vertical direction with finer grid resolutions of $\Delta z=0.5 \mathrm{~mm}$ around the initial bed level and the grid resolution increases gradually away from the initial bed level to a maximum grid size of $\Delta z=$ $2 \mathrm{~mm}$ near the top boundary.

The concentration profiles at the four representative instants are shown in Fig. 4(b-d) and the corresponding phase of each panel is indicated in Fig. 4(a). Using the calibrated value of $B=0.22$ without further tuning, the agreements between the model results and measured data were generally good. During the flow reversal (see Fig. 4 (b) and (d)), only a small amount 
of sediment was suspended and a sharp concentration gradient near the bed was observed. On the contrary, during the positive flow peak (see Fig. 4 (c)), significantly more sediment was suspended with a notably thicker suspension layer. Suspended sediment during the negative flow peak (see Fig. 4 (d)) was less than those during the positive flow peak and the model results also matched well with the measured data.

The agreement between the measured sediment concentration and model results can also be examined via the time series of sediment concentration at four representative locations near the bed (Fig. 5). In the pick up layer (e.g., $z=-0.95 \mathrm{~mm}$, see Fig. 5(b)), namely the region below the initially undisturbed bed $(z<0)$, sediment concentration decreased from about 0.6 (concentration of immobile bed) to about 0.25 as free-stream approaches flow peak. Sediment concentration then increased to about 0.6 as flow became weaker and approaches flow reversal. A similar feature repeated again during the wave trough although the sediment concentration only decreased to about 0.33 due to less energetic flow. Similar agreements were observed at $z=$ $-0.35 \mathrm{~mm}$ (see Fig. 5(c)). The model was clearly able to capture the main characteristic in the pick up layer. In the suspension layer, namely the region above the initially undisturbed bed $(z>0)$, the temporal evolution with respect to the pick-up layer reverses. The behavior was qualitatively captured by the model (see Fig. 5(e)). It is noted that the model cannot predict the burst events during the flow reversal (see $t=2-3 \mathrm{~s}$ in Fig. 5(e)), and the burst events may be due to the turbulence-sediment interactions, which may require a turbulence-resolving model to resolve this phenomenon. However, the model was able to reproduce the nearly constant sediment concentration 
occurring around the initially undisturbed bed level $(z \approx 0$, see Fig. $5(\mathrm{~d}))$.

The erosion depth is defined as the distance between the initial bed level to the location where the particle velocity is sufficiently small $\left(u^{s}<5 \mathrm{~mm} / \mathrm{s}\right)$. The modeled temporal evolution of erosion depth is similar to the measured data (Fig. 6), although it was also observed that the model tends to overpredict the erosion depth during positive or negative flow peak by about $0.5 \mathrm{~mm}$. On the contrary, during flow reversal, the model under-predicted the erosion depth by about $1 \mathrm{~mm}$. We like to also point out that a difference of 0.5 to $1 \mathrm{~mm}$ is roughly $2-3$ grain diameters, and also it is similar to the measurement error and our numerical grid size.

The model results of velocity profiles at four different instants are shown in Fig. 7 (solid curves). During the experiment of O'Donoghue and Wright (2004), concurrent measurement of flow velocity was not performed for medium sand cases. However, velocity profiles of another case under the same wave condition for mixed grain sizes with the same $d_{50}$ (X2A7515) were reported and they were also presented in Fig. 7 (symbols). Overall, the model predicted velocity profiles were in good agreement with the measured data. However, the numerical model slightly over-predicted the flow velocity during positive flow peak (see panel (c)). During the negative wave phase, the agreement was very good (see panel (d), (e)). The same simulation for case MA7515 was also carried out for 2DV domain with $L_{x}=0.4 \mathrm{~m}$. The resulting flow field was completely uniform in the streamwise direction and the modeled sediment concentration and flow velocity were almost identical to those obtained from the 1DV simulation (not shown). 


\subsection{Scour Downstream of an Apron}

To further validate the present model for more complicated 2DV flow conditions, we simulated the scour development downstream of an apron. Following the numerical study of Amoudry and Liu (2009), the scour problem was simplified and sketched in Fig. 8. The flow depth is chosen to be $h_{0}=$ $0.15 \mathrm{~m}$, and the initial bed depth is $h_{b}=0.05 \mathrm{~m}$. The length of the domain downstream of the apron is chosen to be $L_{x}=1 \mathrm{~m}$. Stretched grid are used in both the streamwise and vertical directions. In the vertical direction, 300 grid points are used with uniform grid size of $\Delta z=0.5 \mathrm{~mm}$ near and below the initial bed level. The vertical grid size then increases away from the initial bed with $\Delta z_{\max } \approx 1.1 \mathrm{~mm}$ near the top boundary. In the streamwise direction, finer resolution is used around the apron with $\Delta x_{\min }=0.5 \mathrm{~mm}$. The grid size then increases gradually away from the apron with the maximum grid size of $\Delta x_{\max }=2.5 \mathrm{~mm}$ near the downstream end of the domain. A symmetric boundary condition is imposed on the top boundary, and at the step wall and bottom wall, wall boundaries are applied for both the fluid and the sediment velocities and sediment concentration. At the downstream boundary, an outlet boundary condition is used for the velocities of both phases. When the flow is going outward, a zero gradient is imposed for the velocities. Otherwise, it is set to zero. As for the fluid pressure, when the flow is going outward, a constant pressure value is imposed. Otherwise, a zero gradient boundary condition is used. For the inlet boundary (left boundary of the domain), a zero gradient boundary condition is used for the pressure, and a rough-wall log-law velocity profile is imposed for both phases,

$$
\frac{u}{u_{*}}=\frac{1}{\kappa} \ln \left(\frac{30 z}{K_{s}}\right)
$$


where $u_{*}=3.69 \mathrm{~cm} / \mathrm{s}$ is the frictional velocity, $\kappa=0.41$ is the Karman constant, and $K_{s}=2.5 d$ is the bed roughness with the grain size specified as $d=0.25 \mathrm{~mm}$. With sediment specific gravity specified as $s=2.65$, the Shields parameter was estimated to be $\theta=0.34$. The domain is initialized with a flat bed having bed concentration of 0.61 . For the initial fluid and sediment velocities above the bed, they are set to be identical to the inlet velocity, and within the bed, both fluid and sediment velocities are set to be zero.

Fig. 9 shows three snapshots of sediment concentration contour and velocity profiles at four different locations down stream of the apron. At $t=5 \mathrm{~s}$, the development of a scour hole near the inlet can be identified (see Fig. 9(a)). The scour angle and scour depth were both small at this instant. Several small bedforms were observed downstream of the scour hole. At $t=15 \mathrm{~s}$ and $t=45 \mathrm{~s}$, the scour hole became sufficiently large and a return flow was observed within the scour hole (see the velocity profiles in Fig. 9(b),(c)). At $t=15 \mathrm{~s}$, the maximum scour depth was located at $x=0.1 \mathrm{~m}$. At $t=45 \mathrm{~s}$, the maximum scour depth occurred further downstream at $x=0.17 \mathrm{~m}$. Bedforms were also observed to migrate away from the inlet.

According to several experimental/observational studies (e.g., Breusers, 1967; Breusers and Raudkivi, 1991), it is clear that the development of scour hole is rapid at the initial stage, and eventually an equilibrium state can be established. An empirical formulation was suggested to describe the initial rapid development of scour depth (Breusers, 1967),

$$
\frac{\delta_{s}}{h_{0}}=\left(\frac{t}{T_{s}}\right)^{n_{s}},
$$

where $T_{s}$ is a characteristic timescale, and the exponent $n_{s}$ characterizes the 
speed of the scour depth growth at the initial stage. Notice that Eq. (33) only describes the initial development of the scour depth, and the equilibrium scour depth may not be determined from this empirical formula. The equilibrium scour shape is generally independent of the flow velocity and grain size if the Shields parameter is sufficiently larger than the critical Shields parameter. On the other hand, the development of upstream scour angle $\alpha_{s}$ can reach equilibrium rapidly. An empirical formula to describe the development of scour angle is written as (Breusers, 1967),

$$
\alpha_{s}=\alpha_{s 0}\left(1-e^{-t / T_{\alpha s}}\right)
$$

where $T_{\alpha s}$ is the equilibrium timescale of scour angle development and $\alpha_{s 0}$ is the equilibrium scour angle. In Fig. 10, the modeled evolution of these two key scour quantities were compared with the empirical formulas (33) and (34). By using the least-square fit to the modeled scour depth, we observe that the model also reproduced the power law for the initial development of scour depth similar to the empirical formula with $n_{s}=0.54$ and $T_{s}=1100 \mathrm{~s}$. Similar to the model of Amoudry and Liu (2009), our model results gave a value of $n_{s}$ at the high range of the reported value but still can be considered reasonable. For instance, Buchko et al. (1988) reported $n_{s}$ as high as 0.6. The best fit value for the modeled scour angle was $\alpha_{s 0}=-14.55^{\circ}$, and $T_{\alpha s}=15.2 \mathrm{~s}$. Modeled $\alpha_{s 0}$ was within the reported range of observation, $-16^{\circ}<\alpha_{s 0}<-9.5^{\circ}$ (Breusers, 1967).

It is worth mentioning that the predicted scour evolution coefficients are sensitive to many factors, especially the initial condition. Model experiments suggested that the sediment concentration of the initial bed has an impact on the evolution of the scour depth. It was found that a uniform bed con- 
centration rendered a rather fast scour process as compared with the initial condition where the bed concentration was obtained directly from the steady state of $1 \mathrm{DV}$ results. We consider the latter to be more realistic and the results reported here were based on the established bed condition from the 1DV model. Meanwhile, the evolution of scour was also sensitive to the $B$ value in the turbulence modulation model (see Eq. (11)). With a lower value of $B(=0.15)$, numerical experiments showed that the scour process was slightly faster $\left(T_{\alpha s}=14 \mathrm{~s}\right)$, and the characteristic scour angle was slightly larger $\left(\alpha_{s 0}=-15.56^{\circ}\right)$. The development of scour depth was also faster with $T_{s}=785 \mathrm{~s}$ and $n_{s}=0.6$. In addition, since the bedload transport dominated the scour process, the frictional stress model may also play a role. It was found that the scour process was slightly slower (i.e., both $T_{\alpha s}$ and $T_{s}$ were larger) when the coefficient $m$ was reduced from $m=3$ to $m=2$ or the angle of repose $\theta_{f}$ was increased from $28^{\circ}$ to $32^{\circ}$. However, the differences were within $15 \%$.

\section{Momentary bed failure in sheet flow}

Several field observations (Foster et al., 2006; Conley and Inman, 1992) and laboratory experiments (Sleath, 1999; Flores and Sleath, 1998) on waveinduced sediment transport revealed that large horizontal pressure gradients induced by the passage of steep near-breaking or broken waves can destabilize the sand bed and drive momentary bed (shear) failure. Comparing with the well-known sheet flow condition, the observed large transport (or erosion depth) cannot be solely explained by bottom shear stress (or Sheilds parameter) and hence the resulting transport rate is postulated to be enhanced 
by horizontal pressure gradient. This mechanism is often called plug flow (Sleath, 1999) or momentary bed failure (Madsen, 1974).

In the oscillating water tunnel, the horizontal pressure gradient can be expressed by free-stream flow acceleration. In order to quantify the effect of the horizontal pressure gradient, a non-dimensional parameter is used, which is often called the Sleath parameter (Sleath, 1999),

$$
S=\frac{\left|\partial p^{f} / \partial x\right|_{\max }}{\rho^{f}(s-1) g}=\frac{U_{0} \omega}{(s-1) g} .
$$

The last expression converts the horizontal pressure gradient to the flow acceleration, which is only appropriate for the conditions in an oscillatory water tunnel. Madsen (1974) and Sleath (1999) both derived similar criteria to describe the initiation of momentary bed failure using a simple force balance. These criteria give a rather large threshold value of Sleath parameter, typically around 0.33. Later, Foster et al. (2006) reported the first field evidence of momentary bed failure (or plug flow) in Duck, NC and demonstrated its occurrence at a much lower threshold value. They argue that in general the initiation of momentary bed failure must also depend on the Shields parameter. Hence, a combined sheet flow-plug flow mode, which is responsible for a large amount of sediment transport, may occur more commonly than previously believed. More recently, Holway et al. (2012) reported an oscillatory water tunnel experiment using bimodal nylon particles. Due to the low density of nylon particles $\left(\rho^{s}=1170 \mathrm{~kg} / \mathrm{m}^{3}\right)$, the resulting Sleath parameter exceeded 0.36 and momentary bed failure occurred. In addition to the large transport rate obtained due to momentary bed failure, they observed wavelike instabilities and billows during the transport. These wave-like instabilities may play a major role in the plug flow dynamics and more quantitative 
investigation is needed.

In order to study the role of horizontal pressure gradient in driving momentary bed failure in sheet flow conditions, 2DV numerical investigations were performed. In Section 3.2, the numerical model was validated with medium sand $(d=0.28 \mathrm{~mm})$ for a second-order Stokes wave motionan of O'Donoghue and Wright (2004). Here, we performed an additional simulation using the same medium sand for sinusoidal wave conditions (O'Donoghue and Wright 2004; Case 1 in Table 3). Model results were first validated with the measured data and then a series of numerical experiments with a wide range of Sleath parameter and Shields parameter for two different grain sizes were carried out to investigate momentary bed failure in sheet flow conditions (see Table 3).

To ensure the domain was tall enough to cover the wave boundary layer in different flow conditions, the domain height was set to be $L_{z}=0.5 \mathrm{~m}$, which is larger than that used in Section 3.2. An initial sediment bed depth of $h_{b}=0.075 \mathrm{~m}$ is specified. Similar vertical spatial discretization is used with minimum $\Delta z$ of $0.25 \mathrm{~mm}$ (maximum $\Delta z$ of $4 \mathrm{~mm}$ ), and the total number of grid points in the z-direction is $N_{z}=595$. The streamwise length of the domain is chosen to be $L_{x}=0.4 \mathrm{~m}$, and a uniform grid resolution of $\Delta x=2 \mathrm{~mm}$ with $N_{x}=200$ are used.

\section{1. $1 D V$ and $2 D V$ sheet flow simulations}

Case 1 was a sinusoidal wave with $T=5 \mathrm{~s}$ and $U_{m}=1.5 \mathrm{~m} / \mathrm{s}$ (O'Donoghue and Wright, 2004). The Sleath parameter and Shields parameters for Case 1 and other cases investigated in this section are summarized in Table 3. The 
Shields parameter, $\theta$, is estimated based on peak flow velocity,

$$
\theta=\frac{0.5 f_{w} U_{m}^{2}}{(s-1) g d},
$$

where $f_{w}$ is the wave friction factor, and it is calculated by (Swart, 1974),

$$
f_{w}=\exp \left[5.213\left(\frac{2.5 d}{A}\right)^{0.194}-5.977\right]
$$

where $A=U_{m} T / 2 \pi$ is the semi-excursion length.

Although the simulation was carried out in 2DV, the modeled flow velocity and concentration fields remained nearly homogeneous in the streamwise direction (not shown). In Fig. 11, modeled sediment concentration profiles (averaged over the $\mathrm{x}$-direction) at four instants during the positive wave phase were compared with the measured data of O'Donoghue and Wright (2004). The agreement was generally good. However, we can also observe that in this case, the model over-predicted the suspended sediment concentration during the flow peak (see Fig. 11c). The discrepancy may be due to the parameterization in Eq. (11) of the degree that particles follow the fluid velocity fluctuations. In this study, the empirical coefficient $B$ associated with this parameterization was calibrated for steady sheet flow (Sumer et al., 1996) of fine sand with $d=0.13 \mathrm{~mm}$. The same value was used here for oscillatory flow of medium sand. Meanwhile, another identical simulation was carried out in 1DV, and the model results from the 1DV simulation (also not shown) was nearly identical to the $2 \mathrm{DV}$ results. In summary, the estimated Shields parameter for Case 1 is 2.16, while the Sleath parameter is $S=0.12$, which is much smaller than the critical value of 0.33 . Hence, a regular sheet flow with complete homogeneity in the streamwise direction are expected. 2DV 
model results indeed reproduced homogeneous flow in the streamwise direction, Case 2 (see Table 3) with wave period reduced to $2.1 \mathrm{~s}$ and hence the Sleath parameter increased to $S=0.28$ produced similar sheet flow results.

\subsection{Combined sheet flow and plug flow}

When the oscillatory period was reduced to $T=1.8 \mathrm{~s}$ (see Case 3 in Table 3 ), the resulting Shields parameter is 2.8. Although sheet flow is expected, model results were no longer homogeneous in the streamwise direction. Snapshots of the 2DV sediment concentration field (Fig. 12) showed billows associated with instabilities of the near bed transport layer. The billows had a streamwise length scale of $5 \sim 10 \mathrm{~cm}$. Because the billows enhance mixing, the sheet flow layer thickness is increased considerably to about $3 \mathrm{~cm}$. In Case 3, the Sleath parameter is increased to $S=0.32$ due to a shorter oscillatory period and hence a larger flow acceleration (horizontal pressure gradient). At such large value of Sleath parameter, momentary bed failure or plug flow is expected. The present model indicated that the occurrence of momentary bed failure or plug flow is associated with the formation of the observed instability and billows near the bed.

Although qualitative, the instability and billows observed in the model results seem to be consistent with several field and laboratory observations. For example, Conley and Inman (1992) described their observation of a carpet of tufts (about $O(6) \mathrm{cm}$, see their Fig. 5) under the crest of near-breaking waves in the surf zone. Madsen (1974) reported divers' observations of sediment bed seemingly explode just prior to the passage of the crest of near-breaking waves. As mentioned previously, similar wave-like instabilities under combined sheet flow and plug flow conditions were observed during a more recent 
experiment performed in a small oscillatory flow tunnel at the Naval Research Laboratory (Holway et al., 2012). Video of sheet flow transport with a bed composed of a bimodal distribution of nylon cylinders $\left(\rho^{s}=1170 \mathrm{~kg} / \mathrm{m}^{3}\right)$ having diameters of $0.5 \mathrm{~mm}$ (red) and $1.0 \mathrm{~mm}$ (white) demonstrated the streamwise sorting of surficial grains. Sequence of four (top view) images shown in Fig. 13 demonstrated the development of wave-like instabilities. Although the primary billows were more or less spanwise homogeneous, there were notable smaller 3D features suggesting that instabilities grew rapidly into turbulent features. We note that in the present 2DV Reynolds-averaged simulation, smaller 3D turbulent features are parameterized by turbulent viscosity/diffusivity and only primary 2D billows can be directly resolved.

More quantitative analysis and comparison between 1DV and 2DV simulations were performed to elucidate the effects of momentary bed failure and billows on the transport dynamics. Since the instability and billows of the near bed transport layer are inhomogeneous in the streamwise direction, the 1DV model is not adequate to simulate the process. Therefore, we carried out the same simulation of Case 3 but in a 1DV domain to understand the role of instabilities and billows on the resulting transport. In Fig. 14, the streamwise-averaged profiles of sediment concentration, fluid velocity and sediment flux under flow peak for Case 3 are presented along with those calculated by the 1DV model. The differences between 1DV and 2DV results for Case 3 were evident. In the 2DV results, a large dense mobile layer from $z=-0.019 \sim 0.01 \mathrm{~m}$ was observed, and more sediment was mobilized, which gave considerably large sediment flux (see Fig. 14(c)). On the other hand, in the $1 \mathrm{DV}$ results the region between $z=-0.019 \sim-0.002 \mathrm{~m}$ was immo- 
bile and the overall mobile layer thickness and sediment flux were several times smaller. Laboratory observation and linear stability analysis for light particle transport in steady channel flow (Charru and Mouilleron-Arnould, 2002; Schaflinger et al., 1995) suggest that instability of particle transport layer may occur when the mobile layer thickness is sufficiently large. Their findings are consistent with the model results presented here.

Fig. 15 further contrasts the erosion depth for Case 1 and Case 3. For Case 1, the model result was also compared with the measured data of O'Donoghue and Wright (2004) in Fig. 15(b). The measured maximum erosion depth occurred during the middle of the deceleration but the predicted peak value occurred just slightly after the peak flow. The model slightly under-predicted the maximum erosion depth by about $0.5 \mathrm{~mm}$ but the general agreement was good. For Case 3 where the momentary bed failure occurred (Fig. 15(c)), the temporal evolution and the magnitude of erosion depth were distinctly different from that of Case 1. We observed that the erosion depth reached its maximum immediately after the flow reversal. Clearly, the rapid increase of erosion depth responded directly to the peak horizontal pressure gradient (or free-stream acceleration) at the flow reversal. Similar features have been observed by several other researchers during the occurrence of plug flow (Foster et al., 2006; Liu and Sato, 2005; Sleath, 1999; Flores and Sleath, 1998). It is also evident that the erosion depth in Case $3\left(\delta_{m} \approx 22 \mathrm{~mm}\right)$ was considerably larger than that of Case $1\left(\delta_{m} \approx 6 \mathrm{~mm}\right)$. Clearly, the this major difference in erosion depth is because the Sleath parameter is sufficiently large, although the Shields parameters in Case 1 and Case 3 were similar (see Table 3 ). 


\subsection{Discussion}

Sediment transport rate is the most important physical quantity to be evaluated. The present model can provide total sediment transport rate without the bedload/suspended load assumptions. To quantify how momentary bed failure can enhance the transport rate, additional simulations were carried out. A total number of 24 cases were presented in this study (see Table 3). Two different grain diameters $(d=0.28 \mathrm{~mm}$ and $d=0.2 \mathrm{~mm})$ were considered and these 24 simulations covered Shields parameter in the range of 1.62 to 6.12 and Sleath parameter in the range of 0.12 to 0.39 .

To compare sediment transport rate under sinusoidal wave motion, the half-wave averaged sediment transport rate $Q_{1 / 2}$ is defined as,

$$
Q_{1 / 2}=\frac{2}{T} \int_{0}^{T / 2} \int_{0}^{L_{z}} u^{s} \phi d z d t .
$$

The nondimensional half-wave averaged sediment transport rate is then defined as,

$$
\Psi=\frac{Q_{1 / 2}}{\sqrt{(s-1) g d^{3}}} .
$$

The normalized half-wave averaged transport rate calculated from the 1DV and 2DV models are both shown in Table 3. As long as the momentary bed failure (or plug flow) did not occur, the predicted sediment transport rate between the $1 \mathrm{DV}$ and $2 \mathrm{DV}$ results were very similar. However, when momentary bed failure/plug flow was observed, the resulting transport rate was significantly enhanced in the $2 \mathrm{DV}$ results. It is clear that the instabilities and billows of the transport layer observed in the 2DV simulation played a major role in the enhancement of the sediment transport rate. 
The 2DV results of the normalized half-wave averaged transport rate for the 24 cases in Table 3 are plotted in Fig. 16. For typical sheet flow transport, the Shields parameter can be used to parameterize the half-wave averaged nondimensional sediment transport rate using a power law:

$$
\Psi=M_{0}\left(\theta-\theta_{c}\right)^{N_{0}} .
$$

For the cases without momentary bed failure (pure sheet flow), the best fit values are $M_{0}=5.78$ and $N_{0}=1.65$, which are quite similar to commonly used sheet flow transport formulae (Ribberink, 1998). However, when momentary bed failure in sheet flow occurred, the 2DV results predicted the transport rates that were considerably ( $10 \sim 20$ times) larger than the pure sheet flow cases (see Fig. 16(a)). It is expected that the sediment transport rate for momentary bed failure in sheet flow may not be solely related to the Shields parameter (Nielsen, 2006), and the critical Shields parameter may become irrelevant. As an attempt to describe the sediment transport rate in momentary bed failure, we further fit the sediment transport rate as a function of Shields parameter $\theta$ regardless of critical Shields parameter $\theta_{c}$ (Hanes and Bowen, 1985),

$$
\Psi=M_{1} \theta^{N_{1}} .
$$

The fitted curve from Eq. 41 is shown in Fig. 16(b). For the cases with momentary bed failure, we obtain $M_{1}=20.36$ and $N_{1}=2.5$. Clearly, the resulting non-dimensional transport rate increases rapidly with the Shields parameter. In general, we can expect that the transport rate also depends on the Sleath parameter.

It was previously found that plug flows typically occurred after the sheet 
flow threshold was exceeded (Foster et al., 2006). Hence, the threshold criteria for momentary bed failure, parameterized by $S_{c r}$, must also depend on the Shields parameter. This conjecture is confirmed by the present model (Fig. 17). Model experiments with a range of $\theta$ and $S$ show that momentary bed failure/plug flow were observed at $S_{c r}$ as large as 0.32 in mild sheet flow conditions $(\theta=1.5)$. However, in energetic sheet flow with high $\theta$, momentary bed failure is observed at $S_{c r}$ as low as $0.1-0.2$.

By rearranging the force balance on the block of sediment given in Sleath (1999), the criterion of bed mobilization is proposed as, $|K \cdot \theta+S|>C$, where $K$ and $C$ are coefficients, which may be related to the bed friction coefficient and bed concentration (Foster et al., 2006; Frank et al., 2015). According to the present model results with two different grain sizes $(d=0.2 \mathrm{~mm}$ and $0.28 \mathrm{~mm}$ ), we obtain the dashed curve shown in Fig. 17 and the momentary bed failure occurs when,

$$
|0.053 \theta+S|>0.426
$$

\section{Conclusion}

A multi-dimensional two-phase Eulerian model for sediment transport, called SedFoam, was developed based on the open-source CFD toolbox, OpenFOAM. The mathematical formulations follow the prior two-phase flow approach for sediment transport of Hsu et al. (2004), and several modifications to the numerical solver twoPhaseEulerFoam are introduced to make the model more robust for sediment transport applications. The model was validated for sheet flow in steady channel flow, oscillatory flow, and scour downstream of an apron. 
The validated model was further used to study momentary bed failure (plug flow) in sheet flow when the horizontal pressure gradient (or flow acceleration in U-tube) is sufficiently large. Consistent with limited field and laboratory observation, model results indicate that momentary bed failure is associated with the rapid growth of large erosion depth during flow reversal. Moreover, large erosion depth further leads to instabilities and billows of the near bed transport layer and the resulting transport rate is significantly enhanced. The criteria of momentary bed failure/plug flow was investigated with a range of Shields parameter and Sleath parameter. Our numerical result was consistent with the empirical formulation proposed by Foster et al. (2006) that the threshold value for the initiation of plug flow is lower when the Shields parameter is larger. More detailed experimental investigations, especially regarding the generation of billows via instabilities and their role on the resulting transport rate are warranted. Due to the turbulence-averaging approach, the present model results are sensitive to the closure coefficients associated with the parameterization on turbulence-sediment interactions. The present study calibrated this empirical coefficient with steady sheet flow data of Sumer et al. (1996). However, when applying the model to oscillatory sheet flow, the model tended to over-predict the suspended sediment concentration during peak flow. To further improve the model and carry out fundamental investigations of turbulence-sediment interactions in sediment transport, a 3D turbulence-resolving simulation is needed. Such model extension will also allow direct resolving the evolution and dissipation of billows associated with the momentary bed failure. 


\section{Acknowledgements}

This study was supported by National Science Foundation (CMMI-1135026; OCE-1356855) and Office of Naval Research (N00014-14-1-0586). Dr. Joseph Calantoni was supported under base funding to the Naval Research Laboratory from the Office of Naval Research. Numerical simulations were carried out on MILLS at the University of Delaware. Simulations also leverage computing resource provided by Extreme Science and Engineering Discovery Environment (XSEDE) (TG-OCE100015). We gratefully acknowledge developers involved in OpenFOAM, which is the foundation of the new solver SedFoam presented in this report. Dr. Xiao Yu provided many useful suggestions during the model development. SedFoam is publicly available via Community Surface Dynamics Modeling System (CSDMS) model repository maintained by GitHub: https://github.com/csdms-contrib/twophaseeulersedfoam

\section{References}

Amoudry, L., Hsu, T.-J., Liu, P. L.-F., 2005. Schmidt number and nearbed boundary condition effects on a two-phase dilute sediment transport model. Journal of Geophysical Research: Oceans (1978-2012) 110 (C9), - .

Amoudry, L., Liu, P.-F., Jul. 2009. Two-dimensional, two-phase granular sediment transport model with applications to scouring downstream of an apron. Coastal Engineering 56 (7), 693-702.

Amoudry, L. O., Liu, P. L.-F., 2010. Parameterization of near-bed processes under collinear wave and current flows from a two-phase sheet flow model. Continental Shelf Research 30 (13), 1403-1416. 
Bakhtyar, R., Barry, D. A., Yeganeh-Bakhtiary, A., Li, L., Parlange, J.-Y., Sander, G., 2010. Numerical simulation of two-phase flow for sediment transport in the inner-surf and swash zones. Advances in water resources 33 (3), 277-290.

Balachandar, S., Eaton, J. K., 2010. Turbulent dispersed multiphase flow. Annual Review of Fluid Mechanics 42, 111-133.

Breusers, H., 1967. Time scale of two-dimensional local scour. Proc. 12th IAHR Congress, Ft. Collins 3, 275-282.

Breusers, H., Raudkivi, A. J., 1991. Scouring. Balkema Rotterdam.

Buchko, M., Kolkman, P., Pilarczyk, K., Hydraulics, D., 1988. Investigation of local scour in cohesionless sediments using a tunnel-model. Delft Hydraulics.

Calantoni, J., Puleo, J. A., Jan. 2006. Role of pressure gradients in sheet flow of coarse sediments under sawtooth waves. Journal of Geophysical Research 111 (C1), C01010.

Calantoni, J., Thaxton, C. S., Mar. 2008. Simple power law for transport ratio with bimodal distributions of coarse sediments under waves. Journal of Geophysical Research 113 (C3).

Carnahan, N. F., Starling, K. E., 1969. Equation of state for nonattracting rigid spheres. The Journal of Chemical Physics 51 (2), 635-636.

Charru, F., Mouilleron-Arnould, H., 2002. Instability of a bed of particles sheared by a viscous flow. Journal of Fluid Mechanics 452, 303-323. 
Chen, C. P., Wood, P. E., Jun. 1985. A turbulence closure model for dilute gas-particle flows. Canadian Journal of Chemical Engineering 63 (3), 349360.

Chen, X., Li, Y., Niu, X., Chen, D., Yu, X., Nov. 2011. A two-phase approach to wave-induced sediment transport under sheet flow conditions. Coastal Engineering 58 (11), 1072-1088.

Cheng, Z., Hsu, T.-J., Aug. 2014. A multi-dimensional two-phase Eulerian model for sediment transport-TwophaseEulerSedFoam (version 1.0). Tech. Rep. CACR-14-08, Center for Applied Coastal Research, University of Delaware.

Chou, Y.-J., Fringer, O. B., Oct. 2010. A model for the simulation of coupled flow-bed form evolution in turbulent flows. Journal of Geophysical Research 115 (C10), C10041.

Coleman, N. L., Jun. 1970. Flume studies of the sediment transfer coefficient. Water Resour. Res. 6 (3), 801-809.

Conley, D. C., Inman, D. L., Jun. 1992. Field observations of the fluidgranular boundary layer under near-breaking waves. Journal of Geophysical Research 97 (C6), 9631-9643.

Danon, H., Wolfshtein, M., Hetsroni, G., Mar. 1977. Numerical calculations of two-phase turbulent round jet. International Journal of Multiphase Flow $3(3), 223-234$. 
Ding, J., Gidaspow, D., Apr. 1990. A bubbling fluidization model using kinetic theory of granular flow. American Institute of Chemical Engineers Journal 36 (4), 523-538.

Dong, P., Zhang, K., 1999. Two-phase flow modelling of sediment motions in oscillatory sheet flow. Coastal Engineering 36 (2), 87-109.

Dong, P., Zhang, K., 2002. Intense near-bed sediment motions in waves and currents. Coastal Engineering 45 (2), 75-87.

Drake, T. G., Calantoni, J., 2001. Discrete particle model for sheet flow sediment transport in the nearshore. Journal of Geophysical Research: Oceans 106 (C9), 19859-19868.

Drew, D. A., 1983. Mathematical modeling of two-phase flow. Annual Review of Fluid Mechanics 15 (1), 261-291.

Ergun, S., 1952. Fluid flow through packed columns. Chemical Engineering Progress 48, 89-94.

Flores, N. Z., Sleath, J. F., 1998. Mobile layer in oscillatory sheet flow. Journal of Geophysical Research: Oceans (1978-2012) 103 (C6), 1278312793.

Foster, D. L., Bowen, A. J., Holman, R. A., Natoo, P., May 2006. Field evidence of pressure gradient induced incipient motion. Journal of Geophysical Research 111 (C5), C05004.

Frank, D., Foster, D., Sou, I. M., Calantoni, J., Chou, P., 2015. Lagrangian 
measurements of incipient motion in oscillatory flows. Journal of Geophysical Research: Oceans 120 (1), 244-256.

Gidaspow, D., 1994. Multiphase flow and fluidization: continuum and kinetic theory descriptions. Academic press.

Hanes, D. M., Bowen, A. J., 1985. A granular-fluid model for steady intense bed-load transport. Journal of Geophysical Research: Oceans (1978-2012) 90 (C5), 9149-9158.

Heald, J., McEwan, I., Tait, S., 2004. Sediment transport over a flat bed in a unidirectional flow: simulations and validation. Philosophical Transactions of the Royal Society of London A 362, 1973-1986.

Hoffmans, G., Pilarczyk, K., Apr. 1995. Local scour downstream of hydraulic structures. Journal of Hydraulic Engineering 121 (4), 326-340.

Holway, K., Thaxton, C. S., Calantoni, J., 2012. Application of a simple power law for transport ratio with bimodal distributions of spherical grains under oscillatory forcing. Advances in Water Resources 48, 47-54.

Hsu, T.-J., Jenkins, J. T., Liu, P. L.-F., 2004. On two-phase sediment transport: sheet flow of massive particles. Proceedings of the Royal Society of London. Series A: Mathematical, Physical and Engineering Sciences 460 (2048), 2223-2250.

Jacobsen, N. G., Fuhrman, D. R., Fredsoe, J., Nov. 2012. A wave generation toolbox for the open-source CFD library: Openfoam. International Journal for Numerical Methods in Fluids 70 (9), 1073-1088. 
Jenkins, J., Savage, S., 1983. A theory for the rapid flow of identical, smooth, nearly elastic, spherical particles. Journal of Fluid Mechanics 130, 187-202.

Johnson, P. C., Jackson, R., 1987. Frictional-collisional constitutive relations for granular materials, with application to plane shearing. Journal of Fluid Mechanics 176, 67-93.

Kranenburg, W. M., Hsu, T.-J., Ribberink, J. S., Oct. 2014. Two-phase modeling of sheet-flow beneath waves and its dependence on grain size and streaming. Advances in Water Resources 72, 57-70.

Lesser, G., Roelvink, J., van Kester, J., Stelling, G., Oct. 2004. Development and validation of a three-dimensional morphological model. Coastal Engineering 51 (89), 883-915.

Li, M., Pan, S., O’Connor, B. A., Dec. 2008. A two-phase numerical model for sediment transport prediction under oscillatory sheet flows. Coastal Engineering 55 (12), 1159-1173.

Liu, H., Sato, S., Mar. 2005. Laboratory study on sheetflow sediment movement in the oscillatory turbulent boundary layer based on image analysis. Coastal Engineering Journal 47 (01), 21-40.

Liu, X., Garcia, M., Jul. 2008. Three-dimensional numerical model with free water surface and mesh deformation for local sediment scour. Journal of Waterway, Port, Coastal, and Ocean Engineering 134 (4), 203-217.

Lun, C., Savage, S., 1987. A simple kinetic theory for granular flow of rough, inelastic, spherical particles. Journal of applied mechanics 54 (1), 47-53. 
Madsen, O. S., 1974. Stability of a sand bed under breaking waves. Coastal Engineering Proceedings; No 14 (1974): Proceedings of 14th Conference on Coastal Engineering, Copenhagen, Denmark, 1974.

Marieu, V., Bonneton, P., Foster, D. L., Ardhuin, F., Sep. 2008. Modeling of vortex ripple morphodynamics. Journal of Geophysical Research 113 (C9), C09007.

Maxey, M. R., Riley, J. J., 1983. Equation of motion for a small rigid sphere in a nonuniform flow. Physics of Fluids 26 (4), 883-889.

Nielsen, P., Jul. 2006. Sheet flow sediment transport under waves with acceleration skewness and boundary layer streaming. Coastal Engineering $53(9), 749-758$.

O’Donoghue, T., Wright, S., 2004. Concentrations in oscillatory sheet flow for well sorted and graded sands. Coastal Engineering 50 (3), 117-138.

Passalacqua, A., Fox, R., Nov. 2011. Implementation of an iterative solution procedure for multi-fluid gas-particle flow models on unstructured grids. Powder Technology 213 (1-3), 174-187.

Revil-Baudard, T., Chauchat, J., Hurther, D., Barraud, P.-A., 2015. Investigation of sheet-flow processes based on novel acoustic high-resolution velocity and concentration measurements. Journal of Fluid Mechanics 767, $1-30$.

Rhie, C., Chow, W., 1983. Numerical study of the turbulent flow past an airfoil with trailing edge separation. AIAA journal 21 (11), 1525-1532. 
Ribberink, J. S., Jul. 1998. Bed-load transport for steady flows and unsteady oscillatory flows. Coastal Engineering 34 (12), 59-82.

Roelvink, D., Reniers, A., van Dongeren, A., van Thiel de Vries, J., McCall, R., Lescinski, J., Nov. 2009. Modelling storm impacts on beaches, dunes and barrier islands. Coastal Engineering 56 (1112), 1133-1152.

Ruessink, B., Kuriyama, Y., 2008. Numerical predictability experiments of crossshore sandbar migration. Geophysical Research Letters 35 (1).

Rusche, H., 2002. Computational fluid dynamics of dispersed two-phase flows at high phase fractions. Ph.D. thesis, Imperial College London (University of London).

Schaeffer, D. G., 1987. Instability in the evolution equations describing incompressible granular flow. Journal of Differential Equations 66 (1), 19-50.

Schaflinger, U., Acrivos, A., Stibi, H., Aug. 1995. An experimental study of viscous resuspension in a pressure-driven plane channel flow. International Journal of Multiphase Flow 21 (4), 693-704.

Sinclair, J., Jackson, R., 1989. Gas-particle flow in a vertical pipe with particle-particle interactions. AIChE Journal 35 (9), 1473-1486.

Sleath, J., Oct. 1999. Conditions for plug formation in oscillatory flow. Continental Shelf Research 19 (13), 1643-1664.

Srivastava, A., Sundaresan, S., 2003. Analysis of a frictional-kinetic model for gas-particle flow. Powder technology 129 (1), 72-85. 
Sumer, B. M., Kozakiewicz, A., Fredsoe, J., Deigaard, R., 1996. Velocity and concentration profiles in sheet-flow layer of movable bed. Journal of Hydraulic Engineering 122 (10), 549-558.

Swart, D., 1974. Offshore sediment transport and equilibrium beach profiles. W.D. Meinema B.V. Delft Hydraulics Laboratory.

Sweby, P. K., Oct. 1984. High resolution schemes using flux limiters for hyperbolic conservation laws. SIAM Journal on Numerical Analysis 21 (5), 995-1011.

Tardos, G. I., 1997. A fluid mechanistic approach to slow, frictional flow of powders. Powder Technology 92 (1), 61-74.

van Rijn, L., Oct. 1984a. Sediment transport, part I: Bed load transport. Journal of Hydraulic Engineering 110 (10), 1431-1456.

van Rijn, L., Nov. 1984b. Sediment transport, part II: Suspended load transport. Journal of Hydraulic Engineering 110 (11), 1613-1641.

van Rijn, L. C., 1984c. Sediment pick-up functions. Journal of Hydraulic Engineering 110 (10), 1494-1502.

Warner, J. C., Sherwood, C. R., Signell, R. P., Harris, C. K., Arango, H. G., 2008. Development of a three-dimensional, regional, coupled wave, current, and sediment-transport model. Computers \& Geosciences 34 (10), 12841306.

Weller, H., 2002. Derivation, modelling and solution of the conditionally averaged two-phase flow equations. Tech. rep., OpenCFD Ltd. 
1069 Wen, C., Yu, Y., 1966. Mechanics of fluidization. In: Chemical Engineering 1070 Progress Symposium Series. Vol. 62. p. 100.

$1071 \mathrm{Yu}$, X., Hsu, T.-J., Hanes, D. M., 2010. Sediment transport under wave 1072 groups: Relative importance between nonlinear waveshape and nonlinear 1073 boundary layer streaming. Journal of Geophysical Research 115 (C2).

${ }_{1074} \mathrm{Yu}$, X., Hsu, T.-J., Jenkins, J. T., Liu, P. L.-F., Nov. 2012. Predictions of 1075 vertical sediment flux in oscillatory flows using a two-phase, sheet-flow 1076 model. Advances in Water Resources 48 (0), 2-17. 


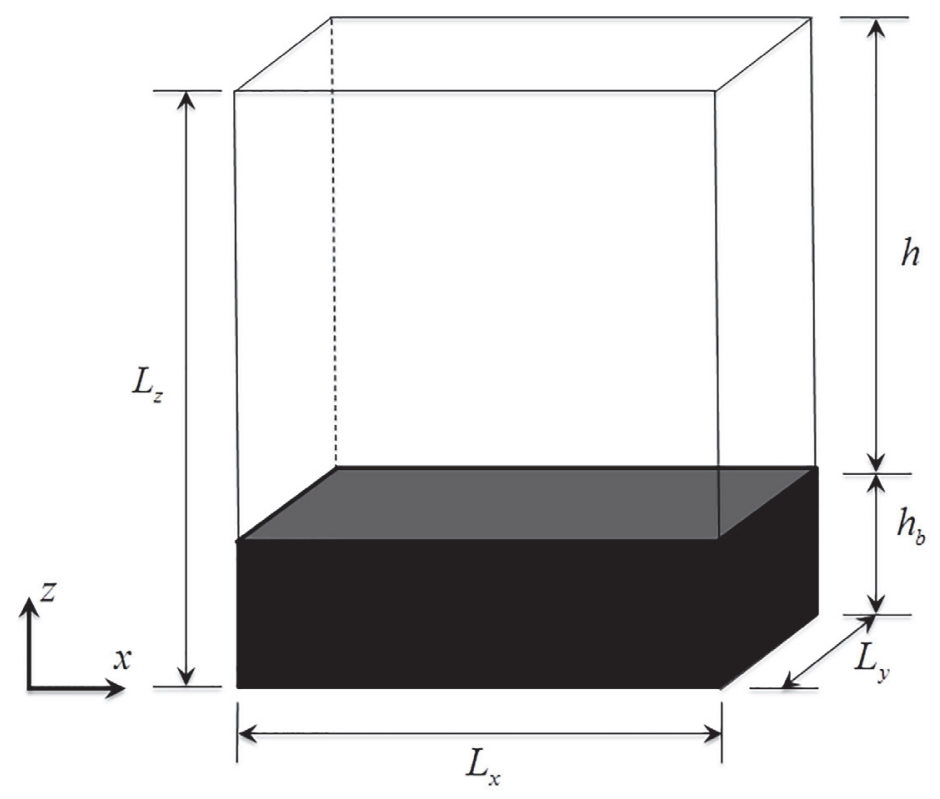

Fig. 2: Sketch of the domain setup. 

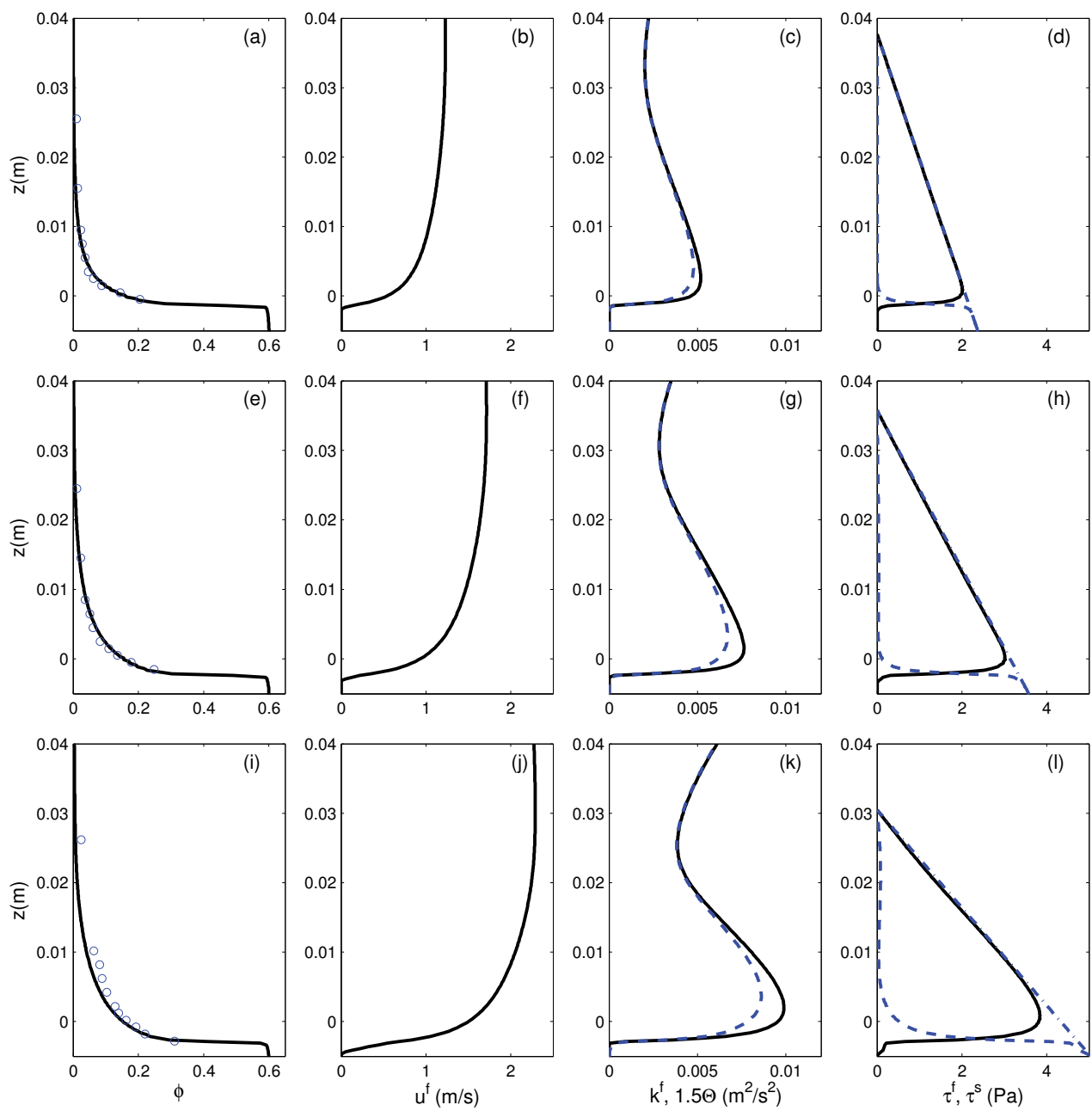

Fig. 3: (a) Comparison of concentration profiles between numerical results (solid curve) and experiment data (circle symbols); (b) fluid velocity profile; (c) fluid turbulent kinetic energy (solid curve) and granular temperature(dashed curve) and (d) fluid shear stress (solid curve), sediment shear stress (dashed curve) and total shear stress (dash-dot curve) for steady sheet flow of at $\theta=1.10$ (Sumer et al., 1996). The corresponding results for $\theta=1.68(\theta=2.2)$ are shown in (e), (f), (g) and (h) ((i), (j), (k) and (l) ), respectively. The bed location $z=0$ is defined as initial bed level. 

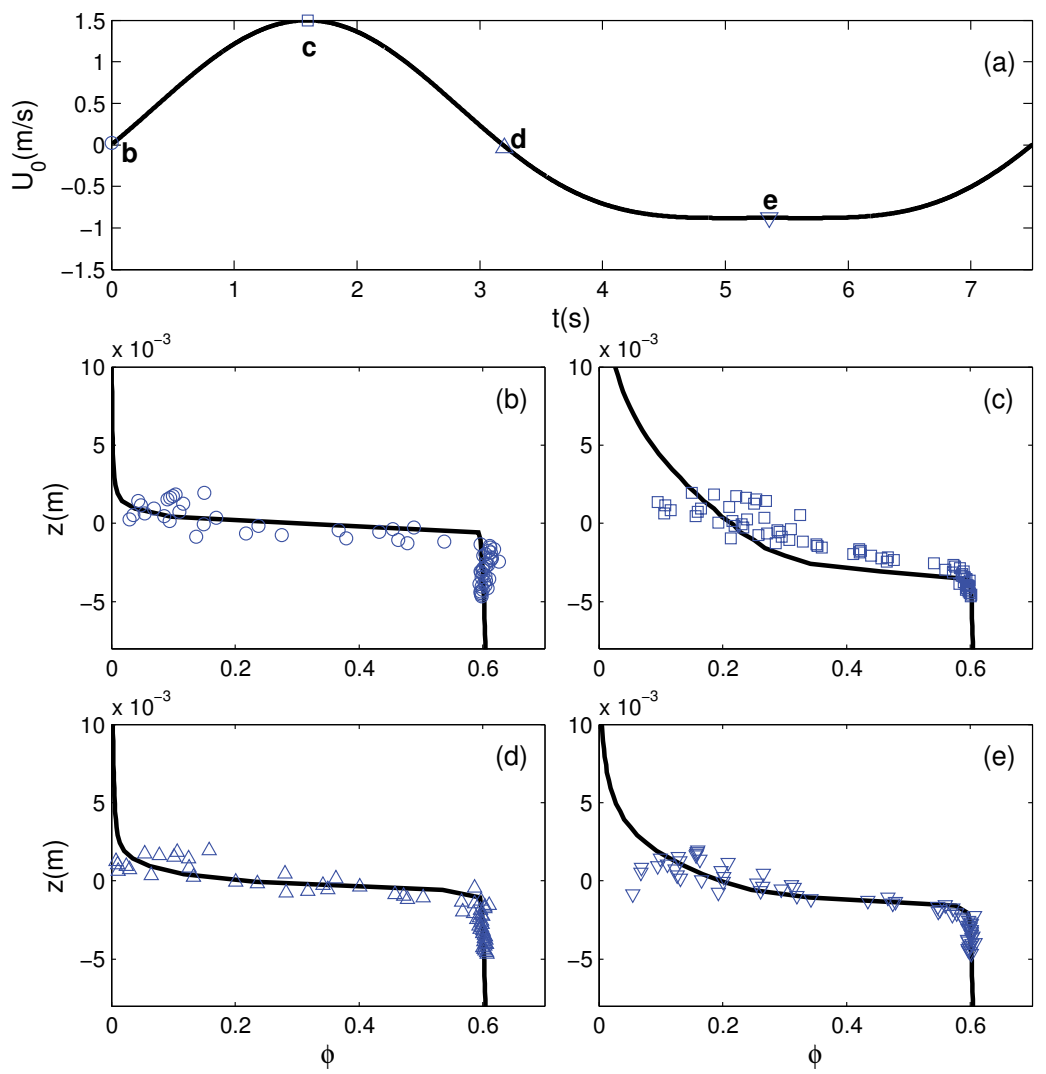

Fig. 4: (a) Time series of free-stream velocity, in which four representative instants are indicated: (b) $t=0$; (c) $t=1.6 \mathrm{~s}$; (d) $t=3.2 \mathrm{~s}$ and (e) $t=5.36 \mathrm{~s}$; The corresponding comparison of sediment concentration profiles between the numerical results (solid curve) and measured data (symbols) from O'Donoghue and Wright (2004) are presented in panel (b), (c), (d) and (e), respectively. 

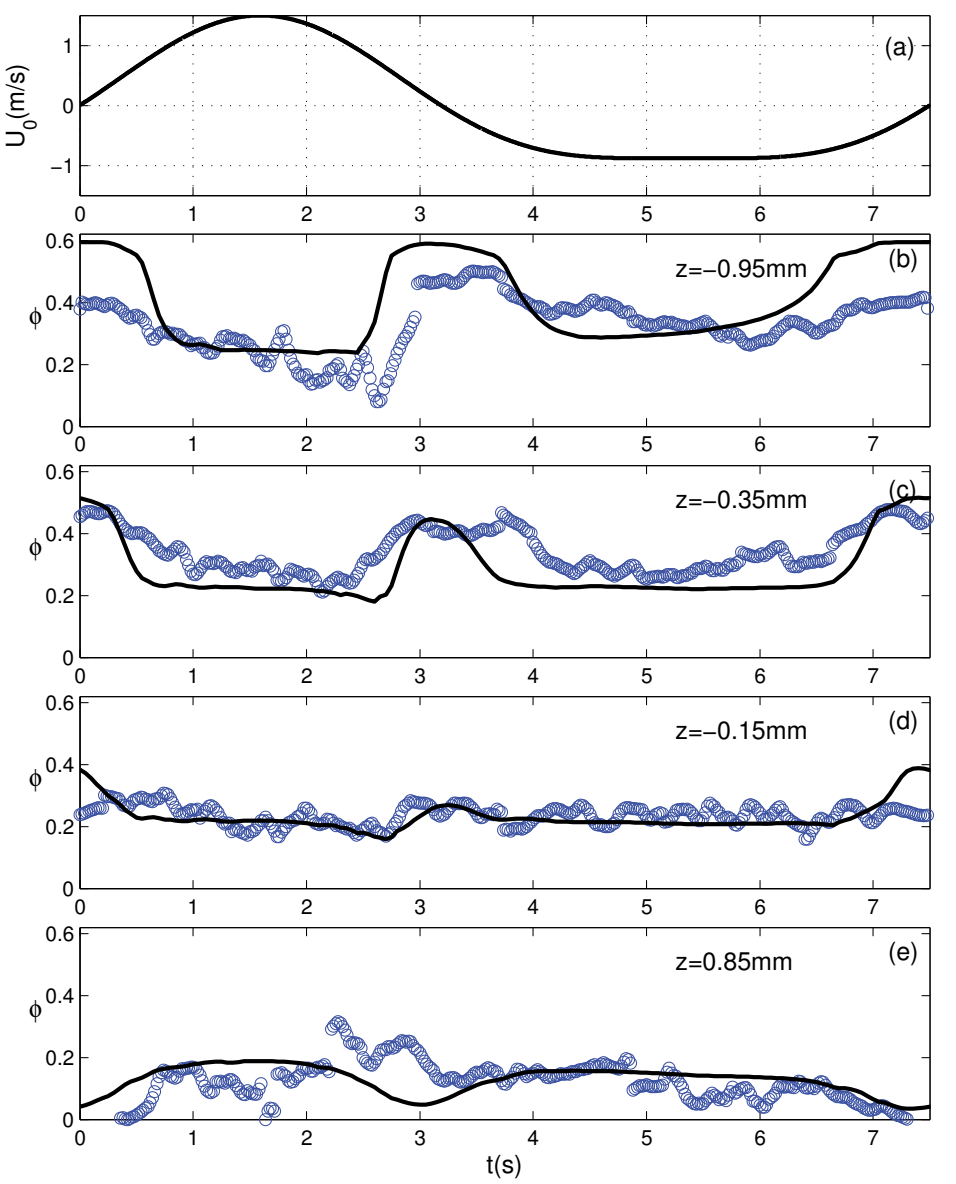

Fig. 5: (a) Time series of free stream velocity driven by Stokes second-order wave motion (MA7515, O'Donoghue and Wright (2004)); The comparisons of concentration time series for sheet flow of medium sand at four different vertical locations are shown in (b) $z=-0.95 \mathrm{~mm}$; (c) $z=-0.35 \mathrm{~mm}$; (d) $z=-0.15 \mathrm{~mm}$ and (e) $z=0.85 \mathrm{~mm}$. 

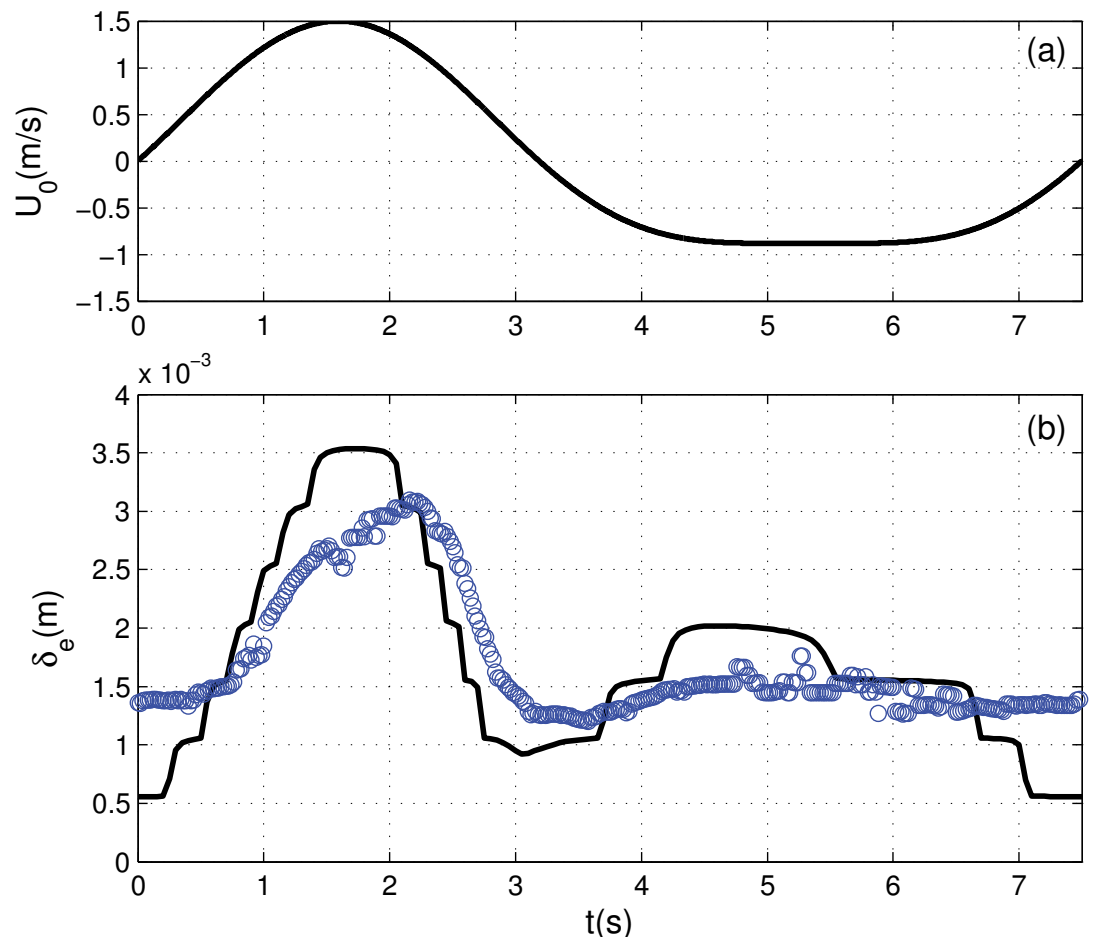

Fig. 6: (a) Time series of free-stream velocity, and (b) Comparison of erosion depth between numerical results (solid curve) and experiment data (symbols) of MA7515 in O'Donoghue and Wright (2004). 

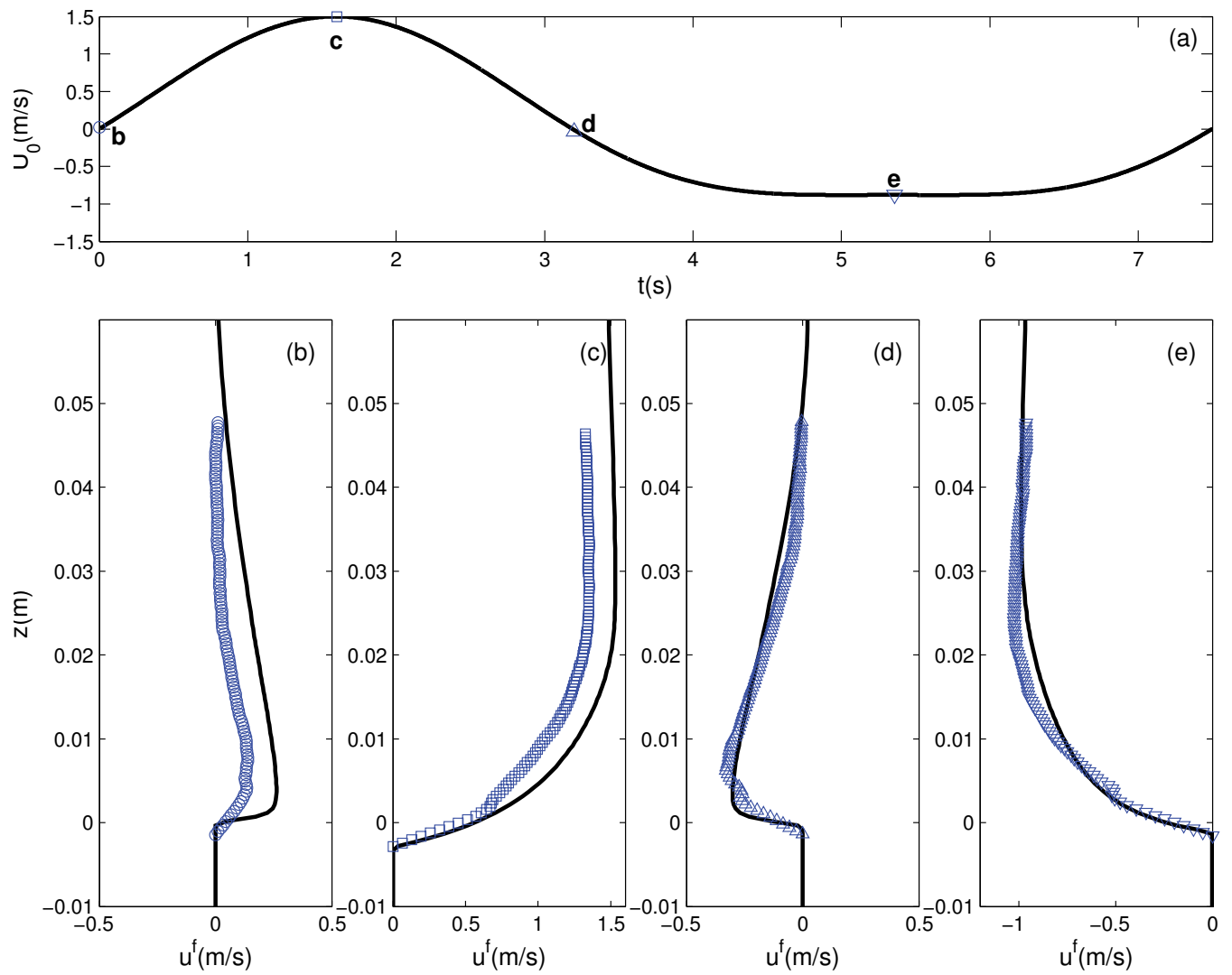

Fig. 7: (a) Time series of free-stream velocity for MA7515 in O'Donoghue and Wright (2004), in which four representative instants are indicated: (b) $t=0$; (c) $t=1.6 \mathrm{~s}$; (d) $t=3.2 \mathrm{~s}$ and (e) $t=5.36 \mathrm{~s}$; The corresponding comparison of fluid velocity profiles between the numerical results (solid curve) and measured data (symbols) at these four instants are shown in panel (b), (c), (d) and (e). 


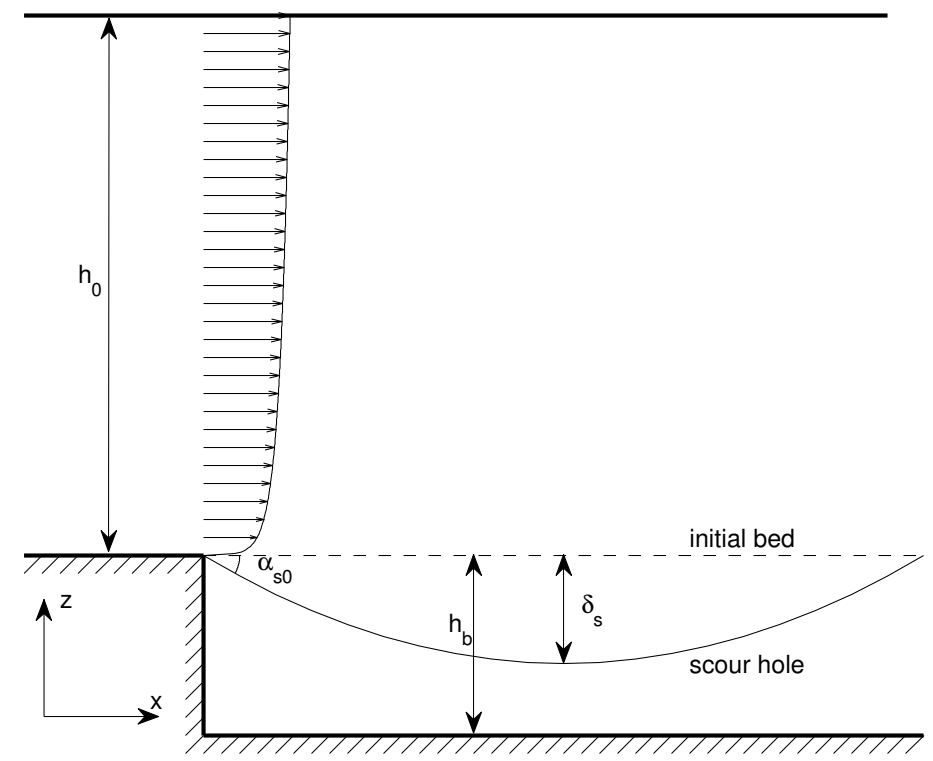

Fig. 8: Sketch of scour downstream of an apron. 

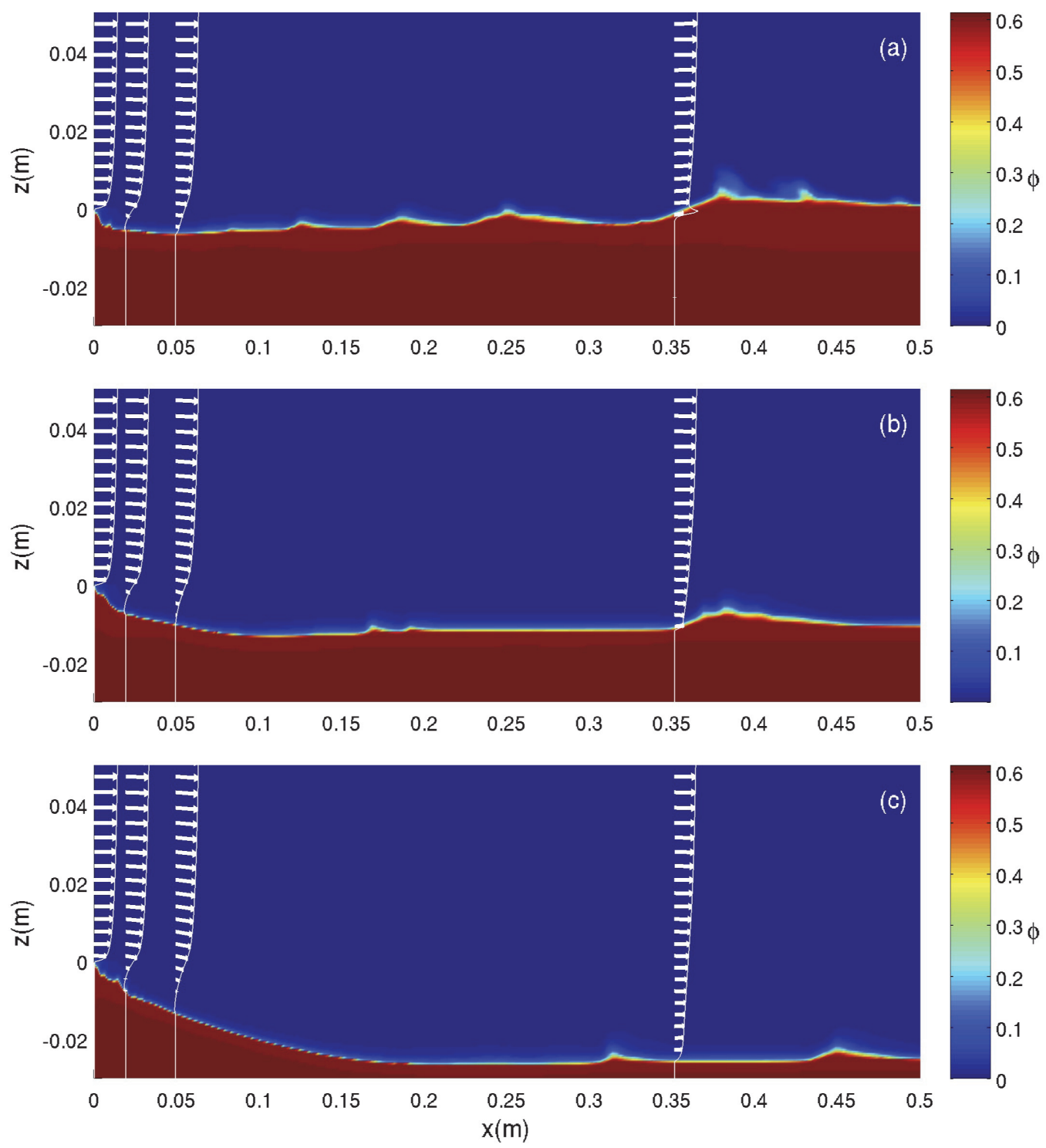

Fig. 9: Velocity profiles at different locations downstream of the apron at (a) $t=5 \mathrm{~s}$, (b) $t=15 \mathrm{~s}$, and (c) $t=45 \mathrm{~s}$; The domain is zoomed in vertical direction to focus on the near-bed processes, and the background is the concentration contour. 

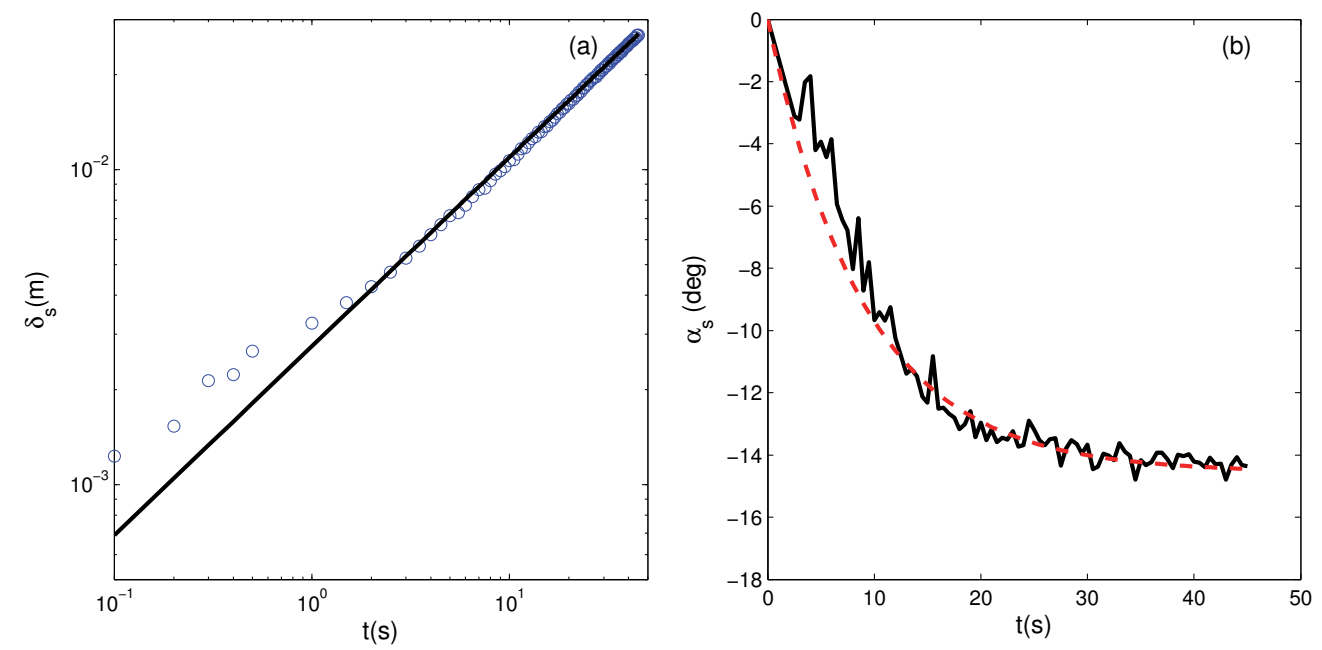

Fig. 10: (a) Evolution of maximum scour depth for model results (symbols) and the empirical formula of Eq. (33) with $T_{s}=1100 \mathrm{~s}$ and $n_{s}=0.54$ (solid line); (b) Evolution of upstream scour angle between model results (dashed curve) and Eq. (34) with $T_{\alpha s}=15.2 \mathrm{~s}$ and $\alpha_{s 0}=-14.55^{\circ}$ (solid curve). 
Table 3: Flow conditions

\begin{tabular}{|c|c|c|c|c|c|c|c|c|}
\hline Cases & $d(\mathrm{~mm})$ & $T(\mathrm{~s})$ & $U_{m}(\mathrm{~m} / \mathrm{s})$ & $\theta$ & $S$ & bed instability & $\Psi_{1 D}$ & $\Psi_{2 D}$ \\
\hline 1 & 0.28 & 5 & 1.5 & 2.16 & 0.12 & no & 19.2 & 19.29 \\
\hline 2 & 0.28 & 2.1 & 1.5 & 2.7 & 0.28 & no & 21.53 & 21.96 \\
\hline 3 & 0.28 & 1.8 & 1.5 & 2.82 & 0.32 & yes & 17.87 & 256.5 \\
\hline 4 & 0.28 & 5 & 2.1 & 3.91 & 0.16 & no & 38.59 & 38.6 \\
\hline 5 & 0.28 & 5 & 2.7 & 6.12 & 0.21 & yes & 95.8 & 1170.8 \\
\hline 6 & 0.28 & 1 & 1 & 1.73 & 0.39 & yes & 8.15 & 67.64 \\
\hline 7 & 0.28 & 1.2 & 1 & 1.62 & 0.32 & no & 7.01 & 7.14 \\
\hline 8 & 0.28 & 1.2 & 1.2 & 2.19 & 0.39 & yes & 11.23 & 117.7 \\
\hline 9 & 0.28 & 1.5 & 1.2 & 2.04 & 0.31 & no & 10.22 & 11.3 \\
\hline 10 & 0.28 & 2.4 & 1.8 & 3.57 & 0.29 & yes & 27.38 & 568.3 \\
\hline 11 & 0.28 & 3 & 2 & 4.05 & 0.26 & yes & 34.43 & 500.3 \\
\hline 12 & 0.28 & 4 & 2 & 3.77 & 0.19 & no & 33.86 & 34.59 \\
\hline 13 & 0.2 & 5 & 1.5 & 2.79 & 0.12 & no & 39.23 & 39.27 \\
\hline 14 & 0.2 & 2.1 & 1.5 & 3.45 & 0.28 & yes & 34.40 & 521.24 \\
\hline 15 & 0.2 & 1.8 & 1.5 & 3.59 & 0.32 & yes & 32.24 & 639.1 \\
\hline 16 & 0.2 & 5 & 2.1 & 5.09 & 0.16 & yes & 97.65 & 3049.3 \\
\hline 17 & 0.2 & 1 & 1 & 2.15 & 0.39 & yes & 11.21 & 103.41 \\
\hline 18 & 0.2 & 1.2 & 1 & 2.03 & 0.32 & yes & 11.17 & 119.4 \\
\hline 19 & 0.2 & 1.2 & 1.2 & 2.76 & 0.39 & yes & 17.96 & 217.9 \\
\hline 20 & 0.2 & 1.5 & 1.2 & 2.58 & 0.31 & yes & 18.34 & 181.29 \\
\hline 21 & 0.2 & 1.2 & 0.9 & 1.7 & 0.29 & no & 9.57 & 9.55 \\
\hline 22 & 0.2 & 3 & 1.5 & 3.14 & 0.19 & no & 39.49 & 39.81 \\
\hline 23 & 0.2 & 5 & 1.8 & 3.86 & 0.14 & no & 65.43 & 65.51 \\
\hline 24 & 0.2 & 6 & 2 & 4.76 & 0.13 & no & 87.99 & 88.05 \\
\hline
\end{tabular}



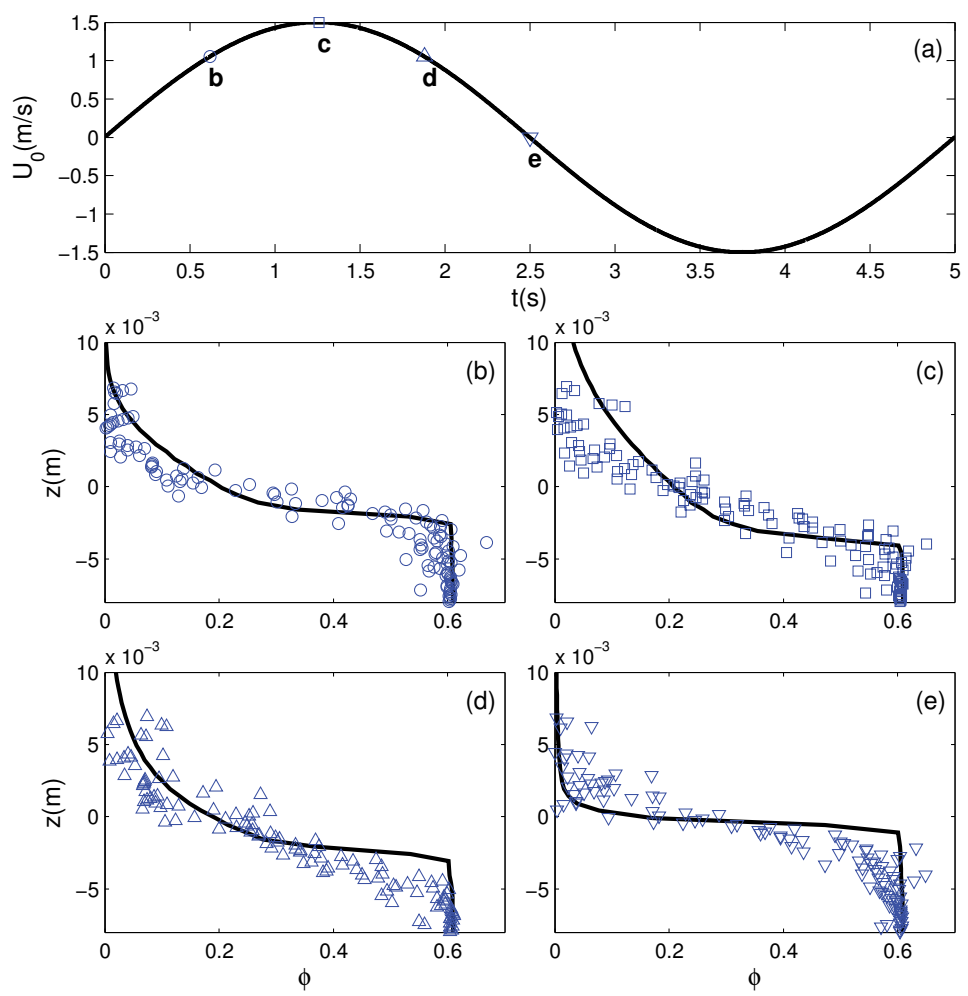

Fig. 11: (a) Time series of free-stream velocity, in which four representative instants are indicated: (b) $t=0$; (c) $t=1.25 \mathrm{~s}$; (d) $t=1.88 \mathrm{~s}$ and (e) $t=2.5 \mathrm{~s}$; The corresponding comparison of sediment concentration profiles between the numerical results (solid curve) and measured data (symbols) at these four instants for Case 1 (see Table 3 ) are shown in panel (b), (c), (d) and (e). 

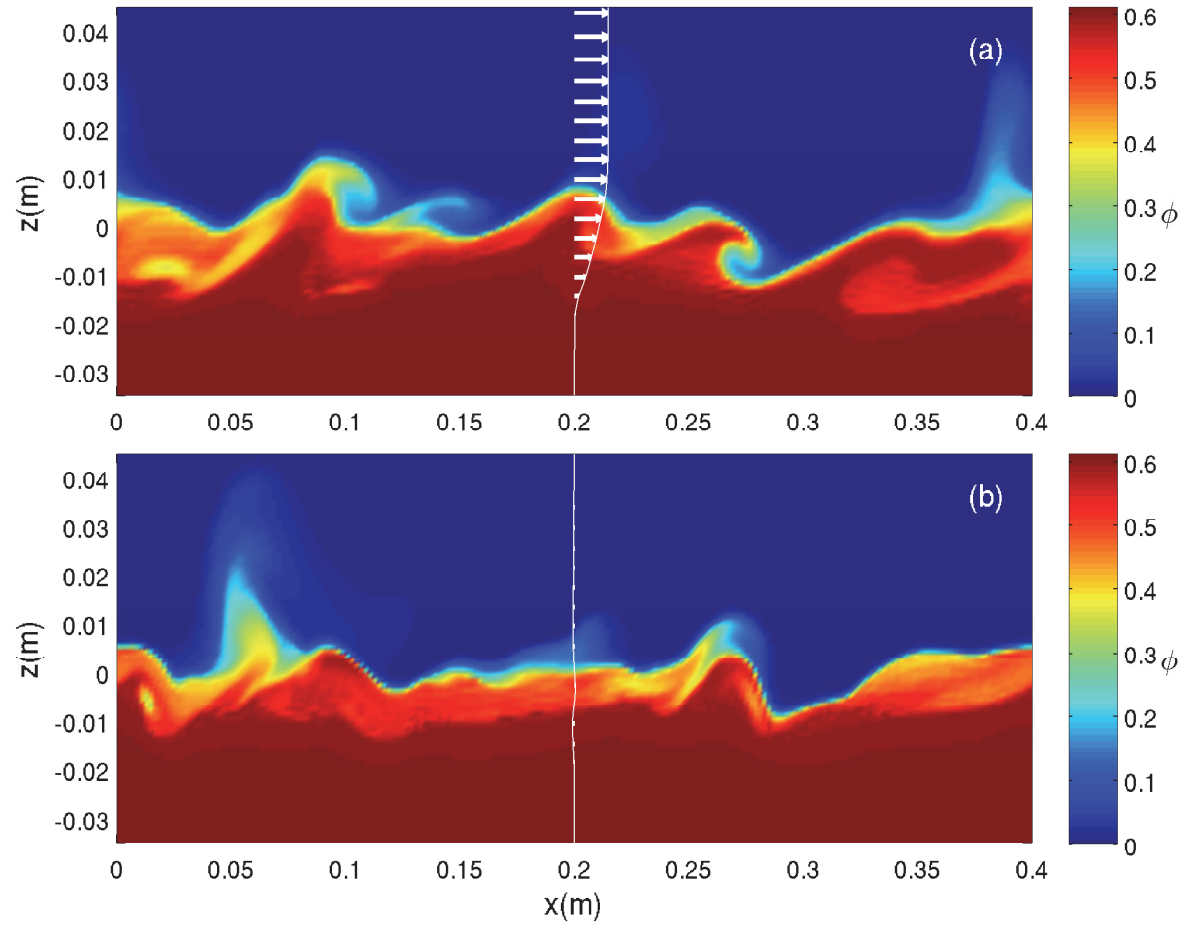

Fig. 12: Snapshot of concentration field at (a) flow peak $(t=0.45 \mathrm{~s})$ and (b) flow reversal $(t=0.9 \mathrm{~s}$ ) for Case 3 (see Table 3 ); white arrows denotes the plane-averaged velocity vectors. 


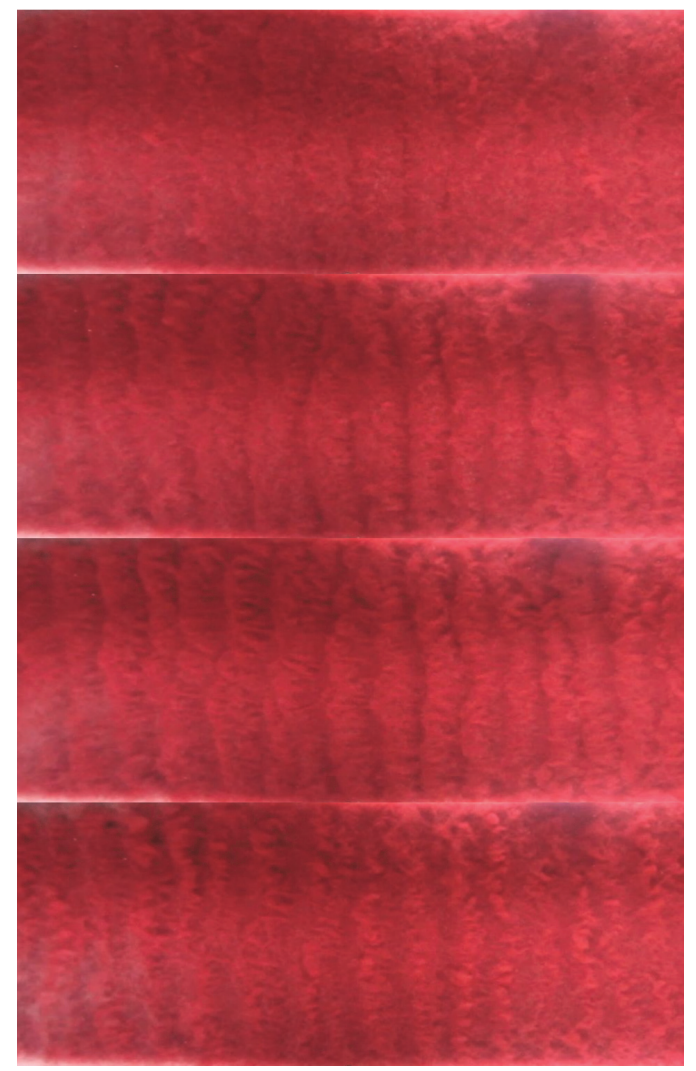

Fig. 13: Sequence of four images (top to bottom) highlighting the development of instabilities and billows under combined sheet flow and plug flow conditions in a small oscillatory flow tunnel during half a wave cycle (Holway et al., 2012). The streamwise length (spanwise width) of the field of view in each snapshot is $64 \mathrm{~cm}(25 \mathrm{~cm})$. 

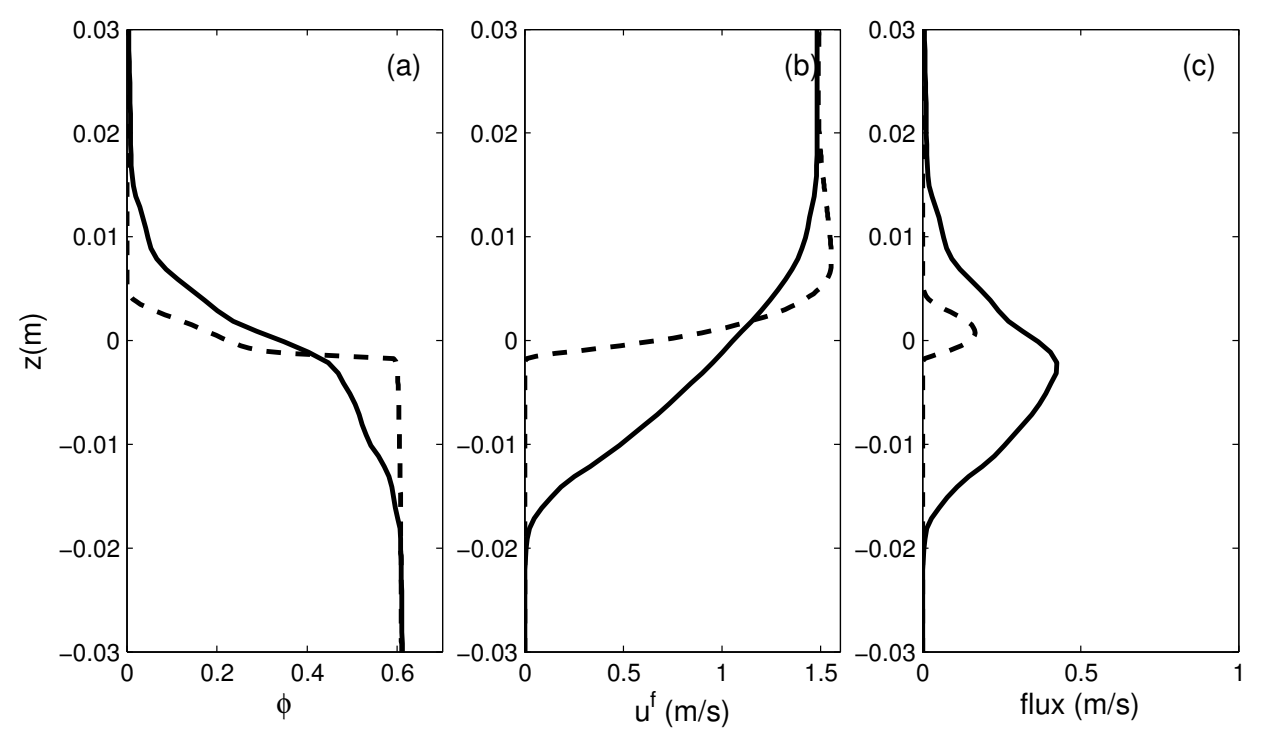

Fig. 14: Comparison of (a) sediment concentration profiles, (b) fluid velocity profiles and (c) sediment flux profiles of 1DV (dashed curves) and 2DV (solid curves) for Case 3 at flow peak. 

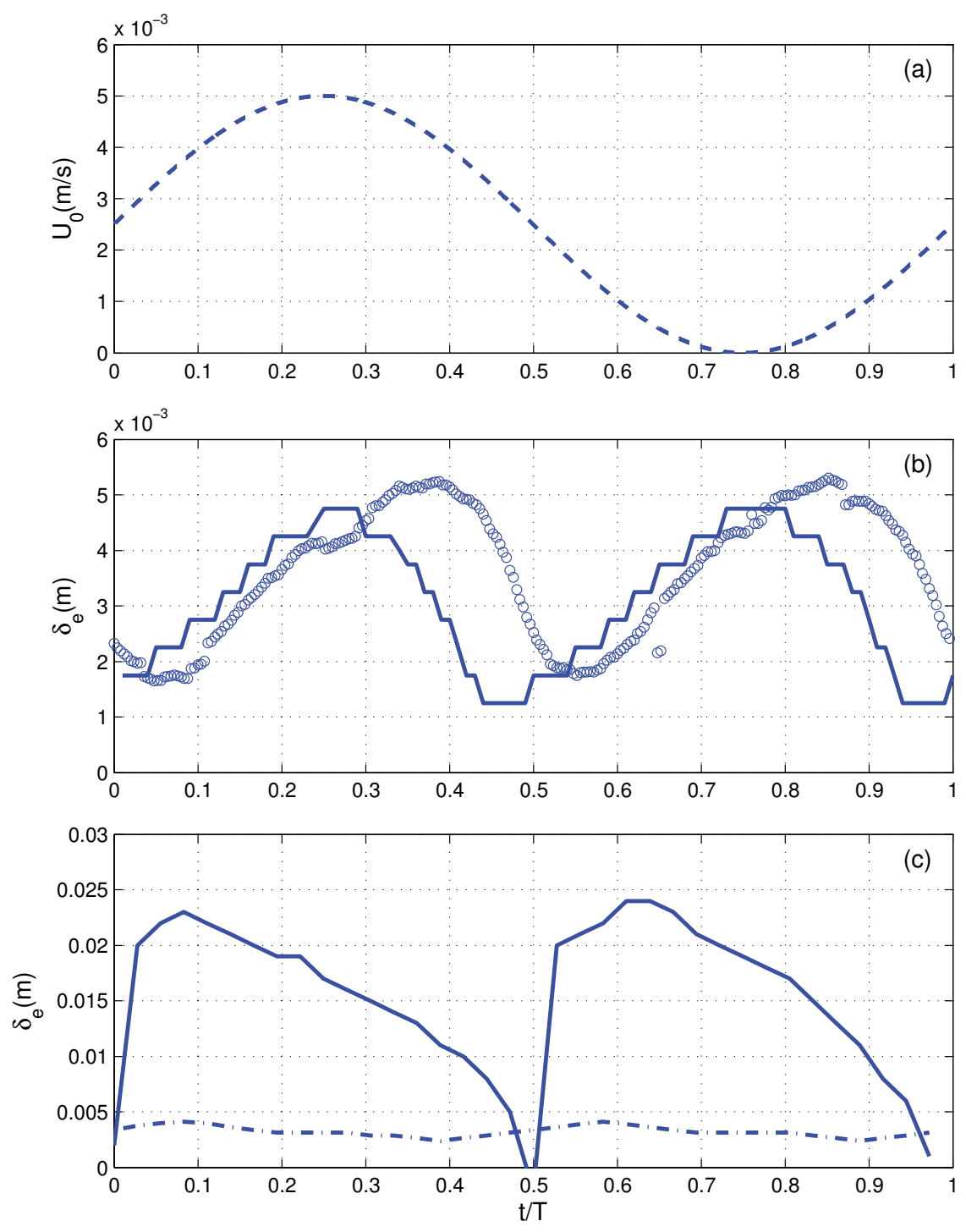

Fig. 15: Time series of free-stream velocity (panel (a)) and numerical results of the erosion depth (solid curves) for (b) Case 1 and (c) Case 3; In panel (b), the circle symbols represent measured data from O'Donoghue and Wright (2004). In panel (c), the dash-dot curve is the $1 \mathrm{DV}$ result. 

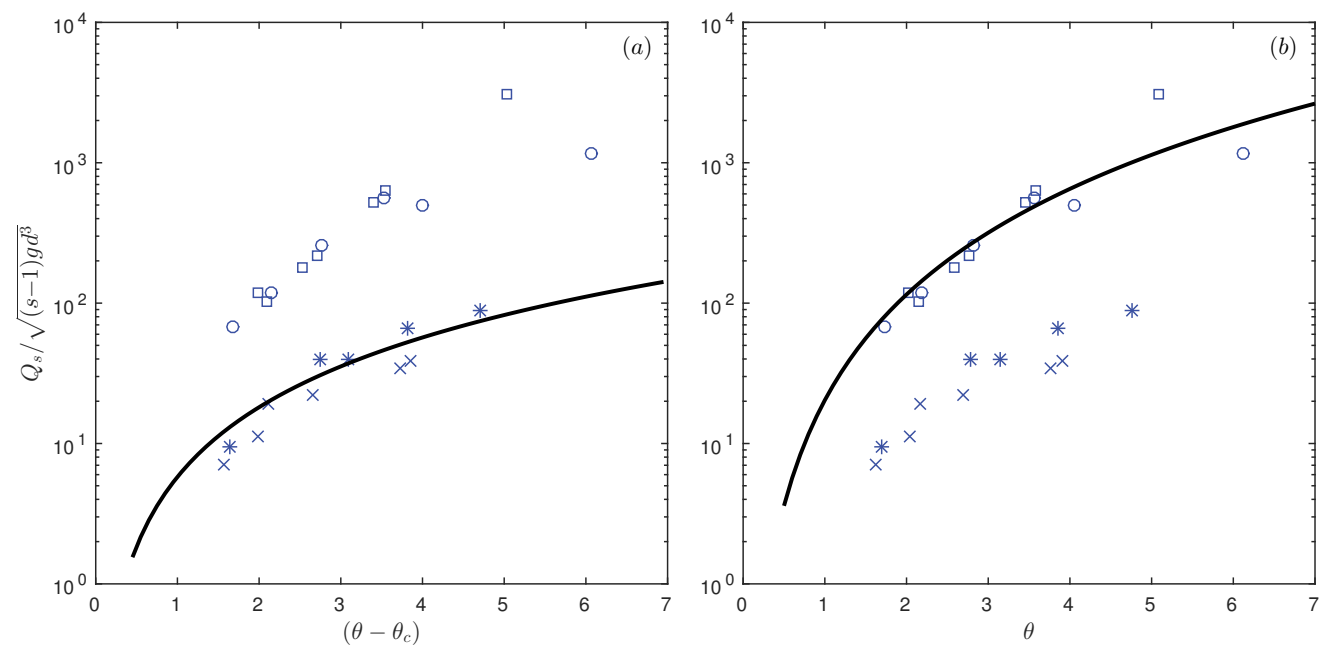

Fig. 16: Panel (a) shows the normalized half-wave-averaged transport rate in terms of Shields parameter $\theta . \quad \times: 2 \mathrm{D}$ pure sheet flow of $d=0.28 \mathrm{~mm} ; 0$ : $2 \mathrm{D}$ sheet flow with momentary bed failure of $d=0.28 \mathrm{~mm} ; *$ : 2D pure sheet flow of $d=0.2 \mathrm{~mm}$; $\square$ : 2D sheet flow with momentary bed failure of $d=0.2 \mathrm{~mm}$; The solid curve shows the best-fit power law (Eq. 40) for pure sheet flow predicted by the present model $\left(M_{0}=5.78, N_{0}=1.65\right)$ where $\theta_{c}=0.05$ is the critical shields parameter. By using Eq. 41, the fitted curve for the sediment transport rate for momentary bed failure (solid curve) is shown in panel (b), which corresponds to $M_{1}=20.36$ and $N_{1}=2.5$ in Eq. 41 . 


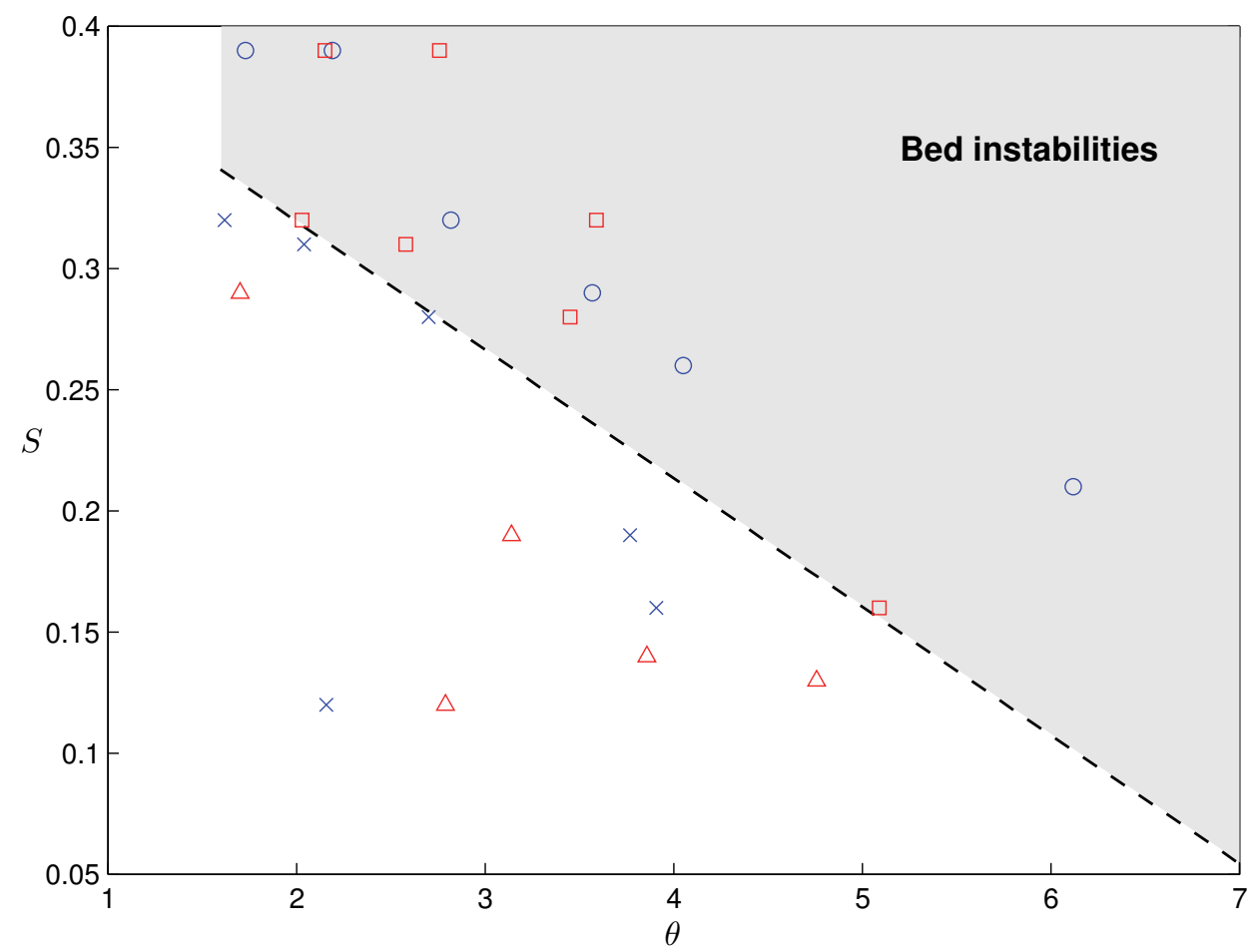

Fig. 17: Schematic map of sheet flow with momentary bed failure in terms of Sleath parameter $S$ and Shields parameter $\theta . \times$ : sheet flows of $d=0.28 \mathrm{~mm}$; $:$ momentary bed failure of $0.28 \mathrm{~mm} ; \triangle$ : sheet flows of $d=0.2 \mathrm{~mm}$; $\square$ : momentary bed failure of $0.2 \mathrm{~mm}$; dashed curve: proposed criteria for the occurrence of momentary bed failure (Eq. (42)). 